\title{
Characterization of some projective subschemes by locally free resolutions
}

\author{
Uwe Nagel
}

\begin{abstract}
A locally free resolution of a subscheme is by definition an exact sequence consisting of locally free sheaves (except the ideal sheaf) which has uniqueness properties like a free resolution. The purpose of this paper is to characterize certain locally Cohen-Macaulay subschemes by means of locally free resolutions. First we achieve this for arithmetically Buchsbaum subschemes. This leads to the notion of an $\Omega$-resolution and extends the main result of Chang in 6 . Second we characterize quasi-Buchsbaum subschemes by means of weak $\Omega$-resolutions. Finally, we describe the weak $\Omega$-resolutions which belong to arithmetically Buchsbaum surfaces of codimension two. Various applications of our results are given.
\end{abstract}

Dedicated to the memory of Wolfgang Vogel

\section{Contents}

1. Introduction 1

2. Preliminaries 4

$\begin{array}{lll}3 . & \text { q-presentations } & 7\end{array}$

4. $\quad$ Surjective-Buchsbaum modules 11

5. Arithmetically Buchsbaum subschemes 14

\begin{tabular}{lll}
\hline 6. Quasi-Buchsbaum subschemes & 23
\end{tabular}

$\begin{array}{lll}\text { 7. } & \text { Surfaces in } \mathbb{P}^{4} & 27\end{array}$

References 30

\section{Introduction}

Since free resolutions have been invented by Hilbert they have proved to be very useful in algebraic geometry and commutative algebra. Indeed, the minimal free resolution of a subscheme reflects its geometric properties (cf., for example, [13], 14], 22) as well as its algebraic properties. For example, the minimal free

2000 Mathematics Subject Classification. Primary 14M05, 14B15; Secondary 13H10, 13D25.

(C)0000 (copyright holder) 
resolution can be used to characterize certain arithmetically Cohen-Macaulay subschemes of projective space. In this paper we want to establish some characterization of non-arithmetically Cohen-Macaulay subschemes by considering more general resolutions.

Let $\mathbb{P}^{n}$ denote the projective space over a field $K$ and let $X \subset \mathbb{P}^{n}$ be a projective subscheme of codimension $c$ with a minimal free resolution

$$
0 \rightarrow \mathcal{F}_{s} \rightarrow \mathcal{F}_{s-1} \rightarrow \ldots \rightarrow \mathcal{F}_{1} \rightarrow \mathcal{J}_{X} \rightarrow 0
$$

where $\mathcal{J}_{X}$ is the ideal sheaf of $X \subset \mathbb{P}^{n}$ and the sheaves $\mathcal{F}_{i}$ are direct sums of line bundles on $\mathbb{P}^{n}$. Due to the Auslander-Buchsbaum formula we know that it holds $s \geq c$ and that $X$ is arithmetically Cohen-Macaulay if and only if $s=c$. We would like to have exact sequences of length $c$ also in the case where $X$ is not arithmetically Cohen-Macaulay. Thus we have to replace the direct sums of line bundles $\mathcal{F}_{i}$ by more general sheaves. But we also want to have a uniqueness property like for minimal free resolutions. This motivates the following concept.

Definition 1.1. An exact sequence of sheaves on $\mathbb{P}^{n}$

$$
0 \rightarrow \mathcal{E}_{s} \rightarrow \mathcal{E}_{s-1} \rightarrow \ldots \rightarrow \mathcal{E}_{1} \rightarrow \mathcal{J}_{X} \rightarrow 0
$$

is said to be a locally free resolution of the subscheme $X \subset \mathbb{P}^{n}$ if

(i) the sheaves $\mathcal{E}_{i}, 1 \leq i \leq s$, are locally free (and possibly satisfy some extra conditions),

(ii) the exact sequence obtained after cancelation of possibly occurring redundant line bundles is unique (up to isomorphisms of exact sequences) among the sequences allowed by (i).

Then the latter sequence is called a minimal locally free resolution of $X$.

Again, we prefer to have locally free resolutions of length $s=c$. Now consider, for example, that the extra condition in (i) is "being direct sums of line bundles". Then we get back the concept of a (minimal) free resolution. In this case $s=c$ iff $X$ is arithmetically Cohen-Macaulay. Moreover, $s=c$ and $\operatorname{rank} \mathcal{E}_{c}=1$ iff $X$ is arithmetically Gorenstein, and $s=c$ and $\operatorname{rank} \mathcal{E}_{1}=c$ iff $X$ is a complete intersection. The purpose of this paper is to extend this list of characterizations of certain arithmetically Cohen-Macaulay subschemes to certain (locally) CohenMacaulay subschemes using locally free resolutions.

Recall that $X$ is arithmetically Cohen-Macaulay if and only if the intermediate cohomology modules $H_{*}^{i}\left(\mathcal{J}_{X}\right), i=1, \ldots, n-c$, vanish. Thus, the next simplest case from a cohomological point of view occurs if all the intermediate cohomology modules are annihilated by the irrelevant maximal ideal $\left(x_{0}, \ldots, x_{n}\right)$. Then $X$ is called quasi-Buchsbaum. An even stronger condition is the property of being arithmetically Buchsbaum. Indeed, $X$ is arithmetically Buchsbaum if and only if $X$ and all its consecutive, sufficiently general hyperplane sections are quasi-Buchsbaum. This is an important concept which has its origin in a negative answer of Vogel 34 to a problem of Buchsbaum. It has been stimulating intensive research (cf., for example, 32] and in particular [33]). A new characterization of arithmetically Buchsbaum subschemes is given by the following result (cf. also Corollary 5.4) where $\Omega_{\mathbb{P}^{n}}^{i}$ denotes the $i$-th exteriour power of the cotangent bundle of $\mathbb{P}^{n}$.

Theorem 1.2. Let $X \subset \mathbb{P}^{n}$ be a subscheme of codimension $c$. Then $X$ is arithmetically Buchsbaum if and only if $X$ admits a locally free resolution of the 
form

$$
0 \rightarrow \mathcal{F}_{c} \rightarrow \ldots \rightarrow \mathcal{F}_{2} \rightarrow \mathcal{F}_{1} \oplus \bigoplus_{j}\left(\Omega_{\mathbb{P}^{n}}^{p_{j}}\left(-e_{j}\right)\right)^{s_{j}} \rightarrow \mathcal{J}_{X} \rightarrow 0
$$

where $\mathcal{F}_{1}, \ldots, \mathcal{F}_{c}$ are direct sums of line bundles and $1 \leq p_{j} \leq n-c, s_{j} \geq 0$.

The locally free resolution in the statement above is called $\Omega$-resolution of $X$. The theorem extends the main result of Chang in $[\mathbf{6}$. from codimension two to arbitrary codimension. It has been proved as Corollary II.3.3 in 25] and independently by C. Walter (unpublished) using different means. Note that in our approach the theorem above is a special case of a more general result characterizing so-called surjective-Buchsbaum subschemes with finite projective dimension as subscheme of an arithmetically Gorenstein scheme (cf. Theorem 5.2). Moreover, we derive necessary conditions for minimal $\Omega$-resolutions and give some applications.

We can also characterize quasi-Buchsbaum schemes by means of a locally free resolution provided their dimension does not exceed the codimension.

Theorem 1.3. Let $X \subset \mathbb{P}^{n}$ be a subscheme of codimension $c \geq \frac{n}{2}$. Then $X$ is quasi-Buchsbaum if and only if $X$ admits a locally free resolution of the form

$$
\begin{aligned}
0 \rightarrow & \mathcal{F}_{c} \rightarrow \ldots \rightarrow \mathcal{F}_{n-c+1} \rightarrow \mathcal{F}_{n-c} \oplus \bigoplus_{j}\left(\Omega_{\mathbb{P}^{n}}^{2(n-c)-1}\left(-e_{n-c . j}\right)\right)^{s_{c-n . j}} \rightarrow \ldots \\
& \rightarrow \mathcal{F}_{2} \oplus \bigoplus_{j}\left(\Omega_{\mathbb{P}^{n}}^{3}\left(-e_{2 . j}\right)\right)^{s_{2 . j}} \rightarrow \mathcal{F}_{1} \oplus \bigoplus_{j}\left(\Omega_{\mathbb{P}^{n}}^{1}\left(-e_{1 . j}\right)\right)^{s_{1 . j}} \rightarrow \mathcal{J}_{X} \rightarrow 0
\end{aligned}
$$

where $\mathcal{F}_{1}, \ldots, \mathcal{F}_{c}$ are direct sums of line bundles and $s_{i . j} \geq 0$.

Again, we give a name to the locally free resolutions occurring in the statement. We call them weak $\Omega$-resolutions. The last result is a special case of Theorem 6.6.

Note that the numbers $e_{i . j}$ and $s_{i . j}$ in the weak $\Omega$-resolution above are uniquely determined by the cohomology of $X$ no matter whether the resolution is a minimal one. Thus, in the case where $X$ is a curve we see that every weak $\Omega$-resolution is an $\Omega$-resolution. This reflects the well-known fact that a curve is arithmetically Buchsbaum if and only if it is quasi-Buchsbaum. The corresponding statement fails in higher dimension. For surfaces in $\mathbb{P}^{4}$ we will characterize the weak $\Omega$-resolution of an arithmetically Buchsbaum subscheme. This result can be applied to conclude from a given weak $\Omega$-resolution that a quasi-Buchsbaum surface is not arithmetically Buchsbaum. For example, consider the smooth rational surface $X \subset \mathbb{P}^{4}$ of degree 10 which can be constructed as degeneracy locus giving rise to the exact sequence

$$
0 \rightarrow\left(\Omega_{\mathbb{P}^{n}}^{3}(-1)\right)^{2} \rightarrow \mathcal{O}(-4) \oplus\left(\Omega_{\mathbb{P}^{n}}^{1}(-3)\right)^{2} \rightarrow \mathcal{J}_{X} \rightarrow 0
$$

(cf. [8], Example B1.15). The second theorem above shows that $X$ is quasiBuchsbaum. However, our results imply that any surface with such a resolution cannot be arithmetically Buchsbaum (cf. Example 7.4).

The paper is organized as follows. In Section 2 we review some results on duality, $k$-syzygies and sheaves having at most one non-vanishing intermediate cohomology. The latter are called Eilenberg-MacLane sheaves. Section 3 describes $q$-presentations. They are the main tool needed to obtain the locally free resolutions above. They have been introduced in local algebra by Auslander and Bridger [3]. We adapt the notion to our purposes and establish the properties we need later on. 
In Section 4 we consider graded modules over a graded Gorenstein $K$-algebra. First we compare the concepts of a surjective-Buchsbaum, Buchsbaum and quasiBuchsbaum module. Second we characterize surjective-Buchsbaum modules of finite projective dimension by the existence of a certain locally free resolution.

In Section 5 we consider arithmetically Buchsbaum subschemes of projective space. Their characterization (Theorem 1.2) is proved there. Then we describe how a free resolution can be obtained from an $\Omega$-resolution, the behaviour of $\Omega$ resolutions under hyperplane sections and consequences for embeddings of abelian varieties. Finally, we derive restrictions for the degree shifts of the vector bundles occurring in a minimal $\Omega$-resolution. This result has as an immediate consequence one of the main results of 15. It says that the Castelnuovo-Mumford regularity of an arithmetically Buchsbaum subscheme is almost determined by its index of speciality. As another application our description of minimal $\Omega$-resolutions has been used in 26 to show that new phenomena occur in the liaison theory of subschemes of codimension $\geq 3$ which do not exist in the case where subschemes of codimension 2 are linked.

In Section 6 we first show that every subscheme of $\mathbb{P}^{n}$ gives rise to an exact sequence

$$
0 \rightarrow \mathcal{E}_{s} \rightarrow \ldots \rightarrow \mathcal{E}_{1} \rightarrow \mathcal{J}_{X} \rightarrow 0
$$

of length $s \leq \max \left\{c, \frac{n}{2}\right\}$ where the sheaves $\mathcal{E}_{i}$ are Eilenberg-MacLane sheaves reflecting the cohomology of $X$ (cf. Theorem 6.1). It follows that every equidimensional Cohen-Macaulay subscheme admits a locally free resolution by EilenbergMacLane bundles. From this result we derive our characterization of quasi-Buchsbaum schemes.

In the final section we consider surfaces in $\mathbb{P}^{4}$. We interpret sequence $(*)$ above as saying that every equidimensional Cohen-Macaulay surface of codimension two is the degeneracy locus of a morphism between Eilenberg-MacLane bundles. We end by establishing a criteria on the weak $\Omega$-resolution of a quasi-Buchsbaum surface $X$ which allows to decide if $X$ is even arithmetically Buchsbaum.

\section{Preliminaries}

In this section we fix notation and collect some results which we will need later on.

Throughout this paper $R=\oplus_{i \in \mathbb{N}} R_{i}$ will denote a graded Gorenstein $K$-algebra where $R_{0}$ is the field $K$ and $R$ is generated by the elements of $R_{1}$. The irrelevant maximal ideal $\oplus_{i>0} R_{i}$ of $R$ is denoted by $\mathfrak{m}_{R}$ or simply $\mathfrak{m}$. Hence $(R, \mathfrak{m})$ is ${ }^{*}$ local in the sense of $[5]$.

If $M$ is a module over the graded ring $R$ it is always assumed to be $\mathbb{Z}$-graded. The set of its homogeneous elements of degree $i$ is denoted by $M_{i}$ or $[M]_{i}$. All homomorphisms between graded $R$-modules will be morphisms in the category of graded $R$-modules, i.e., will be graded of degree zero.

If $M$ is an $R$-module $\operatorname{dim} M$ denotes the Krull dimension of $M$. The symbols $\operatorname{rank}_{R}$ or simply rank are reserved to denote the rank of $M$ in case it has one. For a $K$-module $\operatorname{rank}_{K}$ just denotes the vector space dimension over the field $K$.

There are two types of duals of an $R$-module $M$ we are going to use. The $R$-dual of $M$ is $M^{*}=\operatorname{Hom}_{R}(M, R)$ and the $K$-dual $M^{\vee}=\oplus_{j} \operatorname{Hom}_{K}\left([M]_{j}, K\right)$ where $K$ is considered as a graded module concentrated in degree zero. Both dual modules 
are graded $R$-modules. Note that $R^{\vee}$ is the injective hull of $K^{\vee} \cong K \cong R / \mathfrak{m}$ in the category of graded $R$-modules. If $\operatorname{rank}_{K}[M]_{i}<\infty$ for all integers $i$ then there is a canonical isomorphism $M \cong M^{\vee \vee}$.

Later on we will apply algebraic results in a geometric context. Thus we mention briefly some relations between the algebraic and geometric notions which allow us to switch between the two languages.

Let $\mathcal{F}$ be a sheaf on $Z=\operatorname{Proj}(R)$. The cohomology modules of $\mathcal{F}$ are $H_{*}^{i}(\mathcal{F})=$ $\bigoplus_{t \in \mathbb{Z}} H^{i}(Z, \mathcal{F}(t))$.

There are two functors relating graded $R$-modules and sheaves of modules over $Z$. One is the "sheafification" functor which associates to each graded $R$-module $M$ the sheaf $\tilde{M}$. This functor is exact.

In the opposite direction there is the "twisted global sections" functor which associates to each sheaf $\mathcal{F}$ of modules over $Z$ the graded $R$-module $H_{*}^{0}(\mathcal{F})$. This functor is only left exact. If $\mathcal{F}$ is quasi-coherent then the sheaf $\widetilde{H_{*}^{0}(\mathcal{F})}$ is canonically isomorphic to $\mathcal{F}$. However, if $M$ is a graded $R$-module then the module $H_{*}^{0}(\tilde{M})$ is not isomorphic to $M$ in general. In fact, $H_{*}^{0}(\tilde{M})$ even needs not be finitely generated if $M$ is finitely generated. However, there is the following comparison result (cf. [33]).

Proposition 2.1. Let $M$ be a graded R-module. Then there is an exact sequence

$$
0 \rightarrow H_{\mathfrak{m}}^{0}(M) \rightarrow M \rightarrow H_{*}^{0}(\tilde{M}) \rightarrow H_{\mathfrak{m}}^{1}(M) \rightarrow 0
$$

and for all $i \geq 1$ there are isomorphisms

$$
H_{*}^{i}(\tilde{M}) \cong H_{\mathfrak{m}}^{i+1}(M) .
$$

It follows, for example, that a coherent sheaf $\mathcal{E}$ on $\mathbb{P}^{n}$ is locally free if and only if all modules $H_{*}^{i}(\mathcal{E}), 1 \leq i<n$, have finite length. As usual we will use "vector bundle" and "locally free sheaf" interchangeably. Notice that $H_{*}^{0}(\tilde{M})$ is isomorphic to $H^{0}(M)=\underset{\vec{n}}{\lim } \operatorname{Hom}_{R}\left(\mathfrak{m}^{n}, M\right)$.

Now, we turn to some duality results. Over the Gorenstein ring $R$ duality theory is particularly simple. We denote the index of regularity of a graded ring $A$ by $r(A)$. If $A$ is just the polynomial ring $K\left[x_{0}, \ldots, x_{n}\right]$ then $r(A)=-n$. We will often use the following duality result (cf. [31], [33) without further mentioning.

Proposition 2.2. Let $R$ be a graded Gorenstein ring of dimension $n+1$. Let $M$ be a graded $A$-module where $A$ is a quotient of $R$. Then we have for all $i \in \mathbb{Z}$ natural isomorphisms of graded $R$-modules

$$
H_{\mathfrak{m}_{A}}^{i}(M)^{\vee} \cong \operatorname{Ext}_{R}^{n+1-i}(M, R)(r(R)-1) .
$$

Let $M$ be an $R$-module of dimension $d$. Then

$$
K_{M}=\operatorname{Ext}_{R}^{n+1-d}(M, R)(r(R)-1)
$$

is said to be the canonical module of $M$. It is the module representing the functor $H_{\mathfrak{m}}^{d}\left(M \otimes_{R}{ }_{-}\right)^{\vee}($ cf. [31] $)$. Duality theory also relates the cohomology modules of $M$ and $K_{M}$. Later on we will need the following result of Schenzel [31], Korollar 3.1.3. 
Proposition 2.3. Let $M$ be a graded module over the Gorenstein ring $R$. Suppose that $H_{\mathfrak{m}}^{i}(M)$ has finite length if $i \neq d=\operatorname{dim} M$. Then there are canonical isomorphisms for $i=2, \ldots, d-1$

$$
H_{\mathfrak{m}}^{d+1-i}\left(K_{M}\right) \cong H_{\mathfrak{m}}^{i}(M)^{\vee} .
$$

If the assumption on the cohomology of $M$ in the theorem above is satisfied then $M$ is said to have cohomology of finite length. A graded $R$-module has cohomology of finite length if and only if it is equidimensional and locally Cohen-Macaulay.

Next, we recall some results on $k$-syzygies. If $M$ is an $R$-module then $\operatorname{dim} M \leq$ $\operatorname{dim} R . \quad M$ is said to be maximal if $\operatorname{dim} M=\operatorname{dim} R$. Let $Q$ be the total ring of fractions of $R$. Then an $R$-module $M$ is said to be torsion-free if the natural map $M \rightarrow M \otimes Q$ is injective. The bilinear map $M \times M^{*} \rightarrow R,(m, \varphi) \mapsto \varphi(m)$, induces a natural homomorphism $h: M \rightarrow M^{* *}$. The module $M$ is said to be torsionless if $h$ is injective, and $M$ is said to be reflexive if $h$ is an isomorphism. We want to compare these notions with the following one.

Definition 2.4. Let $M$ be a graded $R$-module. Then $N \neq 0$ is said to be a $k$-syzygy of $M$ if there is an exact sequence of graded $R$-modules

$$
0 \rightarrow N \rightarrow F_{k} \stackrel{\varphi_{k}}{\longrightarrow} F_{k-1} \rightarrow \ldots \rightarrow F_{1} \stackrel{\varphi_{1}}{\longrightarrow} M \rightarrow 0
$$

where the modules $F_{i}, i=1, \ldots, k$, are free $R$-modules.

The $R$-module $N$ is simply called a $k$-syzygy if it is a $k$-syzygy of some $R$ module.

Note that a $(k+1)$-syzygy is also a $k$-syzygy.

Let $\varphi: F \rightarrow M$ be a homomorphism of $R$-modules where $F$ is free. Then $\varphi$ is said to be a minimal homomorphism if $\varphi \otimes i d_{R / \mathfrak{m}}: F / \mathfrak{m} F \rightarrow M / \mathfrak{m} M$ is the zero map in case $M$ is free and an isomorphism in case $\varphi$ is surjective.

In the situation of the definition above $N$ is said to be a minimal $k$-syzygy of $M$ if the morphisms $\varphi_{i}, i=1, \ldots, k$, are minimal. It is uniquely determined up to isomorphism by Nakayama's lemma. If the minimal $k$-syzygy $N$ happens to be free then the exact sequence in the definition above is called a minimal free resolution of $M$.

It follows by [3] that for a finitely generated module over a Gorenstein ring the conditions torsionless, torsion-free and 1-syzygy are all equivalent. The same applies to the conditions reflexive and 2-syzygy. It is more difficult to identify third and higher syzygies. For this we consider the cohomological annihilators $\mathfrak{a}_{i}(M)=\operatorname{Ann}_{R} H_{\mathfrak{m}}^{i}(M)$. Since $R$ is Gorenstein we have $\operatorname{dim} R / \mathfrak{a}_{i}(M) \leq i$ for all integers $i$ where we put $\operatorname{dim} M=-\infty$ if $M=0$. Higher syzygies can be characterized as follows.

Proposition 2.5. Let $M$ be a finitely generated $R$-module. Then the following conditions are equivalent:

(a) $M$ is a k-syzygy.

(b) $\operatorname{dim} R / \mathfrak{a}_{i}(M) \leq i-k$ for all $i<\operatorname{dim} R$.

Moreover, if $k \geq 3$ then conditions (a) and (b) are equivalent to the condition that $M$ is reflexive and $\operatorname{Ext}_{R}^{i}\left(M^{*}, R\right)=0$ if $1 \leq i \leq k-2$. 
Proof. By local duality we know that the annihilators of $\operatorname{Ext}_{R}^{i}(M, R)$ and $H_{\mathfrak{m}}^{\operatorname{dim} R-i}(M)$ coincide. Hence we get

$$
\text { grade } \operatorname{Ext}_{R}^{i}(M, R)=\operatorname{dim} R-\operatorname{dim} R / \mathfrak{a}_{\operatorname{dim} R-i}(M)
$$

and the result follows by [3], Theorem 4.25 and [10], Theorem 3.8.

Following Horrocks [17] a maximal $R$-module $E$ is said to be an EilenbergMacLane module of depth $t, 0 \leq t \leq n+1=\operatorname{dim} R$, if

$$
H_{\mathfrak{m}}^{j}(E)=0 \quad \text { for all } j \neq t \text { where } 0 \leq j \leq n .
$$

An Eilenberg-MacLane module of depth $n+1$ is Cohen-Macaulay, thus a free module if it has finite projective dimension. More generally, a relation between Eilenberg-MacLane modules and syzygy modules is described in the next result which is proved as Proposition I.3.1 and Theorem I.3.9 in 25].

Proposition 2.6. Let $E$ be a module of depth $t \leq n$. Then we have:

(a) If $E$ is an Eilenberg-MacLane module with finite projective dimension then $E^{*}$ is a $(n+2-t)$-syzygy of $H_{\mathfrak{m}}^{t}(E)^{\vee}(1-r(R))$.

(b) If $E$ is reflexive then $E$ is an Eilenberg-MacLane module with finite projective dimension if and only if $E^{*}$ is an $(n+2-t)$-syzygy of a module $M$ of dimension $\leq t-2$. In this case we have

$$
M \cong H_{\mathfrak{m}}^{t}(E)^{\vee}(1-r(R)) .
$$

A sheaf $\mathcal{E}$ on $Z=\operatorname{Proj} R$ is said to be an Eilenberg-MacLane sheaf of depth $t$ $(1 \leq t \leq n)$ if $H_{*}^{t}(\mathcal{E}) \neq 0$ and $H_{*}^{i}(\mathcal{E})=0$ if $i \neq 0, t, n$. Note that in this case $H_{*}^{0}(\mathcal{E})$ is an Eilenberg-MacLane module of depth $t+1$. If $\mathcal{E}$ is in addition locally free we call it an Eilenberg-MacLane bundle.

\section{3. $q$-presentations}

In this section we consider $q$-presentations $(q \in \mathbb{Z})$ and describe some of their properties. They will play a crucial role later on.

These $q$-presentations were first considered by Auslander and Bridger [3] in local algebra. Their uniqueness properties were established by Evans and Griffith 10], 11]. The construction in order to show the existence of 1-presentations is due to Serre [30] (cf. also 23]). These papers work over a local ring. However, we stress the fact that the constructions are also possible over a graded ring.

We slightly modify the notion of $q$-presentations according to our purposes. We focus on local cohomology and state some useful new properties.

Definition 3.1. Let $q$ be an integer with $1 \leq q \leq \operatorname{dim} R$. An exact sequence of finitely generated, graded $R$-modules

$$
0 \rightarrow P \stackrel{\varphi}{\longrightarrow} E \rightarrow M \rightarrow 0
$$

is said to be a q-presentation of $M$ if

(i) $P$ has projective dimension $<q$ and

(ii) $H_{\mathfrak{m}}^{j}(E)=0$ for all $j$ with $\operatorname{dim} R-q \leq j<\operatorname{dim} R$.

It is said to be a minimal $q$-presentation if there does not exist a non-trivial free $R$-module $F$ such that $F$ is a direct summand of $P$ and $E$ and $\varphi$ induces an isomorphism of $F$ onto $F$. 
If the $q$-presentation is not minimal we say that $P$ and $E$ have a common direct free summand.

We begin by recalling that a $q$-presentation "distributes" the local cohomology modules of $M$ among $P$ and $E$ (cf. [26]).

Lemma 3.2. If $0 \rightarrow P \rightarrow E \rightarrow M \rightarrow 0$ is a q-presentation of $M$ then

$$
H_{\mathfrak{m}}^{j}(E) \cong \begin{cases}H_{\mathfrak{m}}^{j}(M) & \text { if } j<\operatorname{dim} R-q \\ 0 & \text { if } \operatorname{dim} R-q \leq j<\operatorname{dim} R\end{cases}
$$

and

$$
H_{\mathfrak{m}}^{j}(P) \cong \begin{cases}0 & \text { if } j \leq \operatorname{dim} R-q \\ H_{\mathfrak{m}}^{j-1}(M) & \text { if } \operatorname{dim} R-q<j<\operatorname{dim} R .\end{cases}
$$

Note that in the definition of a $q$-presentation we have not assumed that $M$ is maximal nor that $P$ is non-trivial. If $P=0$ we say that the $q$-presentation is trivial. Sometimes we know a priori that the modules in the $q$-presentation of $M$ besides $M$ must be maximal.

Lemma 3.3. If $1 \leq q<\operatorname{dim} R-\operatorname{dim} M$ then $M$ admits a trivial $q$-presentation.

If $0 \rightarrow P \rightarrow E \rightarrow M \rightarrow 0$ is a q-presentation then $P$ and $E$ are maximal $R$-modules provided that

(i) $1 \leq \operatorname{dim} R-\operatorname{dim} M \leq q \leq \operatorname{dim} R \quad$ or

(ii) $M$ is maximal and $P \neq 0$.

Proof. The first claim is immediate by the definition.

Now we show the second one. Assume $\operatorname{dim} R-q \leq \operatorname{dim} M<\operatorname{dim} R$. Since $H_{\mathfrak{m}}^{\operatorname{dim} M}(M) \neq 0$ but $H_{\mathfrak{m}}^{\operatorname{dim} M}(E)=0$ we see that $P$ is non-trivial. If $\operatorname{dim} M=$ $\operatorname{dim} R-1$ the exact sequence

$$
0 \rightarrow H_{\mathfrak{m}}^{\operatorname{dim} R-1}(M) \rightarrow H_{\mathfrak{m}}^{\operatorname{dim} R}(P)
$$

shows that $P$ must be maximal. If $\operatorname{dim} M \leq \operatorname{dim} R-2$ then Lemma 3.2 gives

$$
H_{\mathfrak{m}}^{i}(P) \cong \begin{cases}H_{\mathfrak{m}}^{\operatorname{dim}} M(M) \neq 0 & \text { if } i=\operatorname{dim} M+1 \\ 0 & \text { if } \operatorname{dim} M+2 \leq i<\operatorname{dim} R\end{cases}
$$

Suppose $P$ is not maximal. Then we get $\operatorname{dim} P=\operatorname{dim} M+1$ and that $H_{\mathfrak{m}}^{\operatorname{dim} P}(P)^{\vee} \cong$ $H_{\mathfrak{m}}^{\operatorname{dim}} M(M)^{\vee}$ has $\operatorname{dimension} \operatorname{dim} M<\operatorname{dim} P$ contradicting the fact that the dimension of the canonical module of $P$ equals $\operatorname{dim} P$. Thus, the second claim follows in case (i).

If $M$ is maximal and $P \neq 0$ then $P$ must be non-trivial by arguing similarly as above.

Now we turn to the existence of minimal $q$-presentations.

Since $M$ is by assumption finitely generated it is the epimorphic image of a finitely generated free $R$-module. This provides a $(\operatorname{dim} R)$-presentation of $M$ if $M$ has finite projective dimension. In the general case, a $q$-presentation is constructed by induction on $q$. 
Consider the following diagram

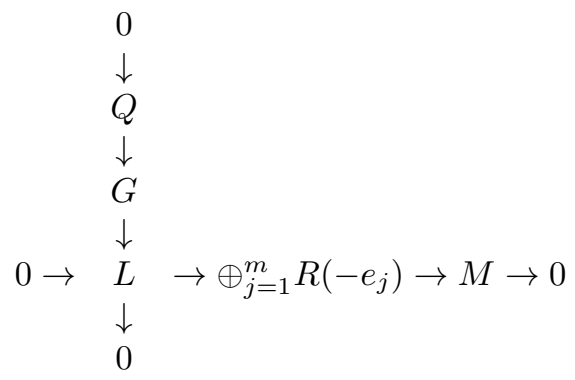

where the row is a minimal free presentation of $M$ and the column is a minimal ( $q-$ 1)-presentation of $L$ provided $q \geq 2$. If $q=1$ we put $Q=0$ and $G=L$. In any case, by composition we get a map $G \rightarrow \oplus_{j=1}^{m} R\left(-e_{j}\right)$ given by elements $g_{1}, \ldots, g_{m} \in G^{*}$. Now we choose elements $g_{m+1}, \ldots, g_{s} \in G^{*}$ such that $\left\{g_{m+1}, \ldots, g_{s}\right\}$ is a minimal basis of $G^{*} /\left(\sum_{j=1}^{m} g_{j} R\left(e_{j}\right)\right)$. Since $L$ is a 1-syzygy we see that $G$ is a 1-syzygy too according to Proposition 2.5. Hence the homomorphism $G \rightarrow \oplus_{j=1}^{s} R\left(-e_{j}\right)$ given by $g_{1}, \ldots, g_{s}$ is injective. Therefore, using the Snake lemma we get an exact commutative diagram

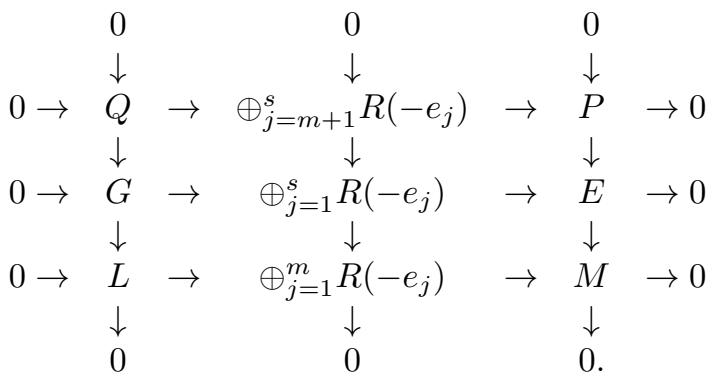

A careful analysis shows that its right-hand column is a minimal $q$-presentation of $M$. This leads to the following result whose detailed proof can be found in 25], Section II.1 (cf. also [10 in the local case).

THEOREM 3.4. A finitely generated $R$-module $M$ admits for every $q$ with $1 \leq$ $q \leq \operatorname{dim} R$ a minimal q-presentation

$$
0 \rightarrow P \rightarrow E \rightarrow M \rightarrow 0
$$

It is uniquely determined up to isomorphisms of exact sequences.

REMARK 3.5. (i) The 1-presentation of $M$ is minimal if and only if the rank of $P$ equals the number of minimal generators of $\operatorname{Ext}_{R}^{1}(M, R)$.

(ii) Contrary to the case $q=1$ it is not clear how one can decide if a given $q$-presentation is minimal if $q \geq 2$. A notable exception is described in Lemma 5.1 later on.

Finally, we would like to discuss the behaviour of $q$-presentations under hyperplane sections. Let $l \in R$ be a general linear form and let $0 \rightarrow P \rightarrow E \rightarrow M \rightarrow 0$ 
be a $q$-presentation of $M$. Suppose depth $M>0$. Then the commutative diagram

$$
\begin{aligned}
& 0 \rightarrow P(-1) \rightarrow E(-1) \rightarrow M(-1) \rightarrow 0 \\
& \downarrow l \quad \downarrow l \quad \downarrow l \\
& 0 \rightarrow \quad P \quad \rightarrow \quad E \quad \rightarrow \quad M \quad \rightarrow 0 .
\end{aligned}
$$

where the vertical maps are multiplication by $l$ provides the exact sequence

$$
0 \rightarrow \bar{P} \rightarrow \bar{E} \rightarrow \bar{M} \rightarrow 0
$$

of $\bar{R}$-modules where $\bar{R}=R / l R$ and ${ }^{-}$denotes the functor $\_\otimes_{R} \bar{R}$. However, this sequence is not the one we are looking for. On the one hand it is in general not a $q$-presentation of $\bar{M}$ (as $\bar{R}$-module). On the other hand we would like to have a $q$-presentation of $H^{0}(\bar{M})$ rather than $\bar{M}$. This is motivated by applications in geometry. For example, if $M$ is reflexive then in general $\bar{M}$ will only be torsion-free whereas $H^{0}(\bar{M})$ is a reflexive $\bar{R}$-module. (This follows by Proposition 2.5). Note also that in case $M$ is an ideal $I \subset R$ the module $H^{0}(\bar{I})$ is just the saturation of $\bar{I}$ in $\bar{R}$. Thus, we will need the following technical result later on.

Lemma 3.6. Let

$$
0 \rightarrow P \rightarrow E \rightarrow M \rightarrow 0
$$

be a q-presentation of $M$ where $1 \leq q \leq \operatorname{dim} R-3$. Let

$$
0 \rightarrow U \rightarrow G \rightarrow H^{0}(\bar{E}) \rightarrow 0
$$

be a q-presentation of $H^{0}(\bar{E})$ as $\bar{R}$-module. Suppose $\operatorname{depth} M>0$. Then there is an $\bar{R}$-homomorphism $\varphi: G \rightarrow H^{0}(\bar{M})$ such that

$$
0 \rightarrow \operatorname{ker} \varphi \rightarrow G \stackrel{\varphi}{\longrightarrow} H^{0}(\bar{M}) \rightarrow 0
$$

is a q-presentation of $H^{0}(\bar{M})$ as $\bar{R}$-module.

Proof. As explained above the assumptions provide an exact sequence

$$
0 \rightarrow \bar{P} \rightarrow \bar{E} \rightarrow \bar{M} \rightarrow 0
$$

which induces the long exact cohomology sequence

$$
0 \rightarrow H^{0}(\bar{P}) \rightarrow H^{0}(\bar{E}) \rightarrow H^{0}(\bar{M}) \rightarrow H^{1}(\bar{P}) \rightarrow \ldots
$$

The assumption on $q$ implies depth $P \geq 4$, thus depth $\bar{P} \geq 3$. It follows (cf. Proposition 2.1) that $H^{0}(\bar{P}) \cong \bar{P}$ and $H^{1}(\bar{P}) \cong H_{\mathfrak{m}}^{2}(\bar{P})=0$. Thus we obtain the following exact commutative diagram where we put $Q=\operatorname{ker} \varphi$ :

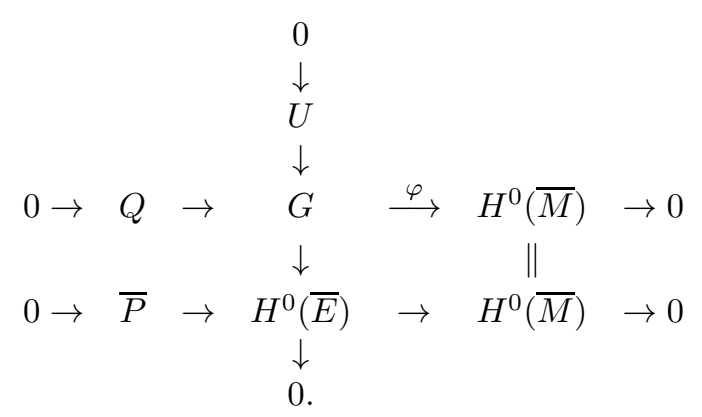

The Snake lemma implies the exact sequence

$$
0 \rightarrow U \rightarrow Q \rightarrow \bar{P} \rightarrow 0
$$


Let $F$ be a minimal free resolution of $P$. Since $l$ is a non-zero divisor of $R$ and $P$ it is well-known that $F_{\bullet} \otimes_{R} \bar{R}$ is a minimal free resolution of $\bar{P}$ as $\bar{R}$-module. Hence $\bar{P}$ as well as $U$ has projective dimension $<q$ as $\bar{R}$-module. Then the so-called Horseshoe lemma implies that this is also true for $Q$. Therefore

$$
0 \rightarrow Q \rightarrow G \rightarrow H^{0}(\bar{M}) \rightarrow 0
$$

is a $q$-presentation as claimed.

\section{Surjective-Buchsbaum modules}

The theory of Buchsbaum modules started from a negative answer of Vogel 34 to a problem posed by Buchsbaum. The concept has been introduced by Stückrad and Vogel. We refer to their monograph 33 for a comprehensive introduction to the subject. Stückrad and Vogel established the so-called surjectivity criterion as a sufficient condition for a module being Buchsbaum (cf. [32], Theorem 1). It gave rise to the notion of a surjective-Buchsbaum module introduced by Yamagishi [36].

In this section we give the definition and discuss the preceding notions. The main result is a characterization of a surjective-Buchsbaum module of finite projective dimension over a Gorenstein ring with the help of its $c$-presentation where $c=\operatorname{dim} R-\operatorname{dim} M$.

As before, we restrict our considerations to the graded situation. We just mention that all the results have analogues for modules over a local ring containing a field. The transfer to this situation is obvious and we omit it.

We begin by recalling some facts of homological algebra. The inclusion soc $M \hookrightarrow$ $H_{\mathfrak{m}}^{0}(M)=\bigcup_{j \geq 1}\left(0:_{M} \mathfrak{m}^{j}\right)$ induces natural homomorphisms of derived functors

$$
\varphi_{M}^{i}: \operatorname{Ext}_{R}^{i}(K, M) \rightarrow H_{\mathfrak{m}}^{i}(M) .
$$

Next we need to consider Koszul complexes. For notation and basic properties of them we refer to [5]. Let $\underline{x}=\left\{x_{1}, \ldots, x_{s}\right\}$ be a minimal basis of the ideal $\mathfrak{m}$. Then the Koszul complexes $K_{\bullet}(\underline{x} ; M)$ and $K^{\bullet}(\underline{x} ; M)$ are up to isomorphism independent of the chosen minimal basis of $\mathfrak{m}$. Thus it makes sense to denote them by $K_{\bullet}(\mathfrak{m} ; M)$ and $K^{\bullet}(\mathfrak{m} ; M)$, respectively, and the homology modules by $H_{i}(\mathfrak{m} ; M)$ and $H^{i}(\mathfrak{m} ; M)$. Since $H_{0}(\mathfrak{m} ; R)=R / \mathfrak{m} \cong K$ this isomorphism lifts to a morphism of complexes from $K_{\bullet}(\mathfrak{m} ; R)$ to a minimal free resolution of $K$. It induces natural homomorphisms

$$
\lambda_{M}^{i}: \operatorname{Ext}_{R}^{i}(K, M) \rightarrow H^{i}(\mathfrak{m} ; M) .
$$

Note that $H^{0}(\mathfrak{m} ; M) \cong 0:_{M} \mathfrak{m}$ can also be embedded into $H_{\mathfrak{m}}^{0}(M)$. The induced natural homomorphisms of derived functors are denoted by

$$
\psi_{M}^{i}: H^{i}(\mathfrak{m} ; M) \rightarrow H_{\mathfrak{m}}^{i}(M) .
$$

Summing up, we have the following commutative diagram for all integers $i$

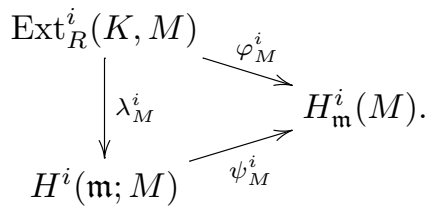

Now we are ready for the following. 
Definition 4.1. The module $M$ is said to be surjective-Buchsbaum if $\varphi_{M}^{i}$ is surjective for all $i \neq \operatorname{dim} M$. It is said to be Buchsbaum if $\psi_{M}^{i}$ is surjective for all $i \neq \operatorname{dim} M$. It is said to be quasi-Buchsbaum if

$$
\mathfrak{m} \cdot H_{\mathfrak{m}}^{i}(M)=0 \text { for all } i \neq \operatorname{dim} M .
$$

It is immediate from the commutative diagram above that a surjective-Buchsbaum module is Buchsbaum and that every Buchsbaum module is quasi-Buchsbaum. Note that these implications are strict in general. However, if $R$ is regular then $K_{\bullet}(\mathfrak{m} ; M)$ is a minimal free resolution of $K$, i.e., $\operatorname{Ext}_{R}^{i}(K, M) \cong H^{i}(\mathfrak{m} ; M)$. Hence, if $R$ is regular then an $R$-module is surjective-Buchsbaum if and only if it is Buchsbaum. Sometimes the three notions are equivalent.

Lemma 4.2. Let $M$ be an $R$-module of depth $t<\operatorname{dim} M$ such that $H_{\mathfrak{m}}^{i}(M)=0$ if $i \neq t, \operatorname{dim} M$. Then $M$ is surjective-Buchsbaum if and only if

$$
\mathfrak{m} \cdot H_{\mathfrak{m}}^{t}(M)=0 .
$$

Proof. The natural map $\varphi_{M}^{t}$ induces an isomorphism

$$
\operatorname{Ext}_{R}^{t}(K, M) \cong \operatorname{Hom}_{R}\left(K, H_{\mathfrak{m}}^{t}(M)\right) .
$$

Hence $\varphi_{M}^{t}$ is surjective if and only if $\mathfrak{m}$ annihilates $H_{\mathfrak{m}}^{t}(M)$.

Note that the last result applies, for example, if $M$ is the coordinate ring of a projective curve.

So far the discussion in this section applies to any graded $K$-algebra $R$. From now on we will make use of our general assumption that $R$ is Gorenstein. Moreover, for the rest of this section we assume that $R$ has positive dimension. We put $n+1=\operatorname{dim} R$ and denote the index of regularity by $r=r(R)$.

Our next goal is to derive a description of the maximal surjective-Buchsbaum modules over $R$ with finite projective dimension. Let

$$
F_{\bullet}: \quad \ldots \rightarrow F_{2} \stackrel{\alpha_{2}}{\longrightarrow} F_{1} \stackrel{\alpha_{1}}{\longrightarrow} F_{0} \stackrel{\alpha_{0}}{\longrightarrow} K \rightarrow 0
$$

be a minimal free resolution of the field $K$. For integers $i$ with $0 \leq i \leq n+1$ we define $G_{i}=\operatorname{coker}\left(\alpha_{n+1-i}^{*}\right)(r-1)$. By local duality we know that $\operatorname{Ext}_{R}^{i}(K, R)=0$ if $i \neq n+1$. Thus $G_{0}(1-r)$ has the following minimal free resolution

$$
0 \stackrel{\alpha_{0}^{*}}{\longrightarrow} F_{0}^{*} \stackrel{\alpha_{1}^{*}}{\longrightarrow} \ldots \rightarrow F_{n}^{*} \stackrel{\alpha_{n+1}^{*}}{\longrightarrow} F_{n+1}^{*} \rightarrow G_{0}(1-r) \rightarrow 0 .
$$

Moreover, the modules $G_{i}$ have the following properties.

Lemma 4.3. Let $0 \leq i \leq n+1$. Then we have:

(a) $H_{\mathfrak{m}}^{0}\left(G_{0}\right) \cong K$ and $G_{0}$ is isomorphic to $K$ if and only if $R$ is regular. Otherwise $G_{0}$ is an Eilenberg-MacLane module.

(b) If $1 \leq i$ then $G_{i}$ is a minimal $i$-syzygy of $G_{0}$ and an Eilenberg-MacLane module of depth $i$ where $H_{\mathfrak{m}}^{i}\left(G_{i}\right) \cong K$ if $i \leq n$.

(c) If $i \leq n$ then $G_{i}^{*}(r-1)$ is a minimal $(n+2-i)$-syzygy of $K$ whereas $G_{n+1}^{*}(r-$ 1) $=R$

(d) $G_{i}$ is surjective-Buchsbaum, indecomposable and has finite projective dimension.

Proof. According to the construction of $G_{0}$ we have $\operatorname{Ext}_{R}^{i}\left(G_{0}, R\right)=0$ if $1 \leq$ $i \leq n$ and $\operatorname{Ext}_{R}^{n+1}\left(G_{0}, R\right) \cong K(r-1)$. Note also that $K$ has finite projective dimension if and only if $R$ is regular. Thus claim (a) follows by local duality. 
Claims (b) and (c) are immediate from the resolutions of $K$ and $G_{0}$ above (cf. also Proposition 2.6).

Suppose $G_{0}$ is decomposable and maximal. Since $K$ is indecomposable it follows by (a) that one of the direct summands of $G_{0}$ must be maximal Cohen-Macaulay, thus free. Hence $G_{0}^{*}$ has a free direct summand contradicting the fact that $G_{0}^{*}$ is a minimal syzygy of the indecomposable module $K(1-r)$. The remaining assertions of (d) follow using Lemma 4.2 .

The next result implies in particular that up to degree shift the modules $G_{i}(0 \leq$ $i \leq n+1)$ are the only indecomposable maximal surjective-Buchsbaum modules of finite projective dimension with the exception of $G_{0}$ in the case where $R$ is regular.

Proposition 4.4. Let $M$ be a maximal module with finite projective dimension. Then the following conditions are equivalent

(a) $M$ is surjective-Buchsbaum.

(b)

$$
M \cong F \oplus \bigoplus_{i=0}^{n} \bigoplus_{j \in \mathbb{Z}} G_{i}^{s_{i j}}(-j)
$$

where $F$ is free and $s_{i j}=\left[h_{\mathfrak{m}}^{i}(M)\right]_{j}$.

Proof. Since $R$ is Gorenstein the $R$-module $M$ has finite projective dimension if and only it has finite injective dimension. Thus the claim follows from the proof of [18], Theorem 3.1.

Observe that the direct sum in the last statement is certainly finite because $M$ is finitely generated by assumption.

The quoted result of Kawasaki has its origin in a theorem of Goto 12] who proved the statement above in the case where $R$ is regular (cf. also [9], Theorem 3.2 ). It is much more difficult to describe the isomorphism classes of maximal modules of finite projective dimension which are Buchsbaum or quasi-Buchsbaum. In particular, there are infinitely many of them if $R$ is not regular (cf. [37])

We need one more preparatory result.

Lemma 4.5. Let

$$
0 \rightarrow P \rightarrow E \rightarrow M \rightarrow 0
$$

be a q-presentation where $1 \leq q \leq \operatorname{dim} R-\operatorname{dim} M$. Then $M$ is surjective-Buchsbaum respectively quasi-Buchsbaum if and only if $E$ has the corresponding property.

Proof. The given $q$-presentation induces for all integers $i$ the following commutative diagram with exact rows:

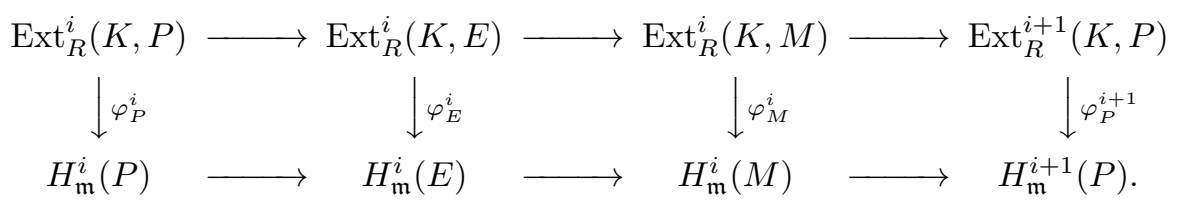

According to the definition of a $q$-presentation we get depth $P \geq \operatorname{dim} R+1-q=$ $n+2-q$. Hence the left- and the right-hand side of the rows in the diagram above vanish if $i \leq n-q$. It follows that for these $i$ the map $\varphi_{E}^{i}$ is surjective if and only if $\varphi_{M}^{i}$ is surjective. Since $\operatorname{dim} M \leq n+1-q$ by assumption and $H_{\mathfrak{m}}^{i}(E)=0$ if $n+1-q \leq i \leq n$ by Lemma 3.2 we obtain that $E$ is surjective-Buchsbaum 
if and only if $M$ is. This also shows the corresponding assertion with respect to the quasi-Buchsbaum property by just considering the lower row in the diagram above.

After these preparations the main result of this section follows easily. Recall that the canonical module of $M$ is denoted by $K_{M}$.

TheOREm 4.6. Let $M$ be an $R$-module such that $c=\operatorname{dim} R-\operatorname{dim} M>0$. Then the following conditions are equivalent:

(a) $M$ is surjective-Buchsbaum with finite projective dimension.

(b) $M$ admits a c-presentation

$$
0 \rightarrow P \rightarrow E \rightarrow M \rightarrow 0
$$

whereby $P$ has projective dimension $c-1, P^{*}$ is a c-syzygy of $K_{M}(1-r)$ and

$$
E \cong F \oplus \bigoplus_{i=0}^{\operatorname{dim} M-1} \bigoplus_{j \in \mathbb{Z}} G_{i}^{s_{i j}}(-j)
$$

where $F$ is free and $s_{i j}=\left[h_{\mathfrak{m}}^{i}(M)\right]_{j}$.

Proof. Let $0 \rightarrow P \rightarrow E \rightarrow M \rightarrow 0$ be a $c$-presentation of $M$. It follows by Lemma 3.3 that $P$ and $E$ must be maximal modules. Hence Lemma 3.2 implies that $P$ is an Eilenberg-MacLane module of depth $n+2-c$ where $H_{\mathfrak{m}}^{n+2-c}(P) \cong$ $H_{\mathfrak{m}}^{n+1-c}(M) \neq 0$ because of $\operatorname{dim} M=n+1-c$. Now the Auslander-Buchsbaum formula gives the claim on the projective dimension and Proposition 2.6 yields that $P^{*}$ is a $c$-syzygy of $H_{\mathfrak{m}}^{n+1-c}(M)^{\vee}(1-r) \cong \operatorname{Ext}_{R}^{c}(M, R)=K_{M}(1-r)$.

Since $P$ has finite projective dimension the same is true for $E$ if and only if $M$ has finite projective dimension.

Thus the asserted equivalence follows by Proposition 4.4 and Lemma 4.5 .

Observe that the module $P$ in the $c$-presentation above is torsion-free but not reflexive. Replacing $P$ by its minimal free resolution we obtain.

Corollary 4.7. Let $M$ be an R-module such that $c=\operatorname{dim} R-\operatorname{dim} M>0$. Then the following conditions are equivalent:

(a) $M$ is surjective-Buchsbaum with finite projective dimension.

(b) $M$ admits a locally free resolution

$$
0 \rightarrow F_{c} \rightarrow \ldots \rightarrow F_{1} \rightarrow F_{0} \oplus \bigoplus_{i=0}^{\operatorname{dim} M-1} \bigoplus_{j \in \mathbb{Z}} G_{i}^{s_{i j}}(-j) \rightarrow M \rightarrow 0
$$

where $F_{0}, \ldots, F_{c}$ are free $R$-modules.

\section{Arithmetically Buchsbaum subschemes}

In this section we show how the methods of the previous sections can be applied in order to study an arithmetically Buchsbaum subscheme of projective space. We begin by considering the $(c-1)$-presentation of an ideal $I \subset R$ because it turns out that it is more useful than the $c$-presentation of $R / I$. Then we obtain a characterization of projective arithmetically Buchsbaum subschemes by means of the so-called $\Omega$-resolution.

Throughout the rest of this paper $I$ will be a homogeneous ideal in the graded Gorenstein $K$-algebra $R$. We put $A=R / I, n+1=\operatorname{dim} R, r=r(R)$ and denote 
the codimension of $I$ by $c$, i.e. $\operatorname{dim} A=n+1-c$. We will always assume that $2 \leq c \leq n$. Otherwise the results become rather trivial.

Our first goal is a minimality criterion for the $(c-1)$-presentation of $I$. In order to unify the statement we say that a free module $F$ is a 0 -syzygy of a module $N$ if there is an epimorphism $\varphi: F \rightarrow N$. F is called a minimal 0 -syzygy if the map $\varphi$ is minimal.

LEMma 5.1. Let

$$
0 \rightarrow P \rightarrow E \rightarrow I \rightarrow 0
$$

be a $(c-1)$-presentation of $I$. Then $P$ is a reflexive Eilenberg-MacLane module of projective dimension $c-2$ and $P^{*}$ is a $(c-1)$-syzygy of $K_{A}(1-r)$.

Moreover, the given $(c-1)$-presentation is minimal if and only if $P^{*}$ is a minimal $(c-1)$-syzygy of $K_{A}(1-r)$.

Proof. Lemma 3.2 provides that $P$ is an Eilenberg-MacLane module of depth $n+3-c$ where $H_{\mathfrak{m}}^{n+3-c} \cong H_{\mathfrak{m}}^{n+1-c}(A)$ if $c \geq 3$. This implies that $P$ is reflexive and has the claimed projective dimension. Proposition 2.6 gives that $P^{*}$ is a a $(c-1)$-syzygy of $K_{A}(1-r)$ if $c \geq 3$. If $c=2$ the last claim is immediate from the given 1-presentation and the minimality assertion follows by Remark 3.5 .

It remains to show the minimality criterion if $c \geq 3$. Assume that the given presentation is not minimal. Then there is a non-trivial free module $F$ such that $P \cong F \oplus Q$ for some module $Q$. Since $Q$ has the same intermediate cohomology as $P, Q^{*}$ is also a $(c-1)$-syzygy of $K_{A}(1-r)$ by Proposition 2.6, i.e. $P^{*}$ is not a minimal $(c-1)$-syzygy of $K_{A}(1-r)$.

Now suppose that the given $(c-1)$-presentation is minimal. We have to show that $P^{*}$ does not have a free direct summand. Dualizing the presentation we obtain the exact sequence

$$
0 \rightarrow R \rightarrow E^{*} \rightarrow P^{*} \rightarrow \operatorname{Ext}_{R}^{1}(I, R) \rightarrow \ldots
$$

But $\operatorname{Ext}_{R}^{1}(I, R) \cong \operatorname{Ext}_{R}^{2}(A, R) \cong H_{\mathfrak{m}}^{n-1}(A)^{\vee}(1-r)=0$ because $\operatorname{dim} A=n+1-c \leq$ $n-2$. Thus we see: if $P^{*}$ has a free direct summand then $E^{*}$ has this direct summand too, contradicting the assumed minimality of the given presentation of $I$.

Using the modules $G_{i}$ defined before Lemma 4.3 we state the main result of this section.

THEOREM 5.2. With the notation above the following conditions are equivalent

(a) $A=R / I$ is surjective-Buchsbaum of finite projective dimension.

(b) I admits an exact sequence

$$
0 \rightarrow F_{c} \rightarrow \ldots \rightarrow F_{2} \rightarrow F_{1} \oplus \bigoplus_{i=1}^{n+1-c} \bigoplus_{j \in \mathbb{Z}}\left(G_{i}(-j)\right)^{t_{i j}} \rightarrow I \rightarrow 0
$$

where $F_{1}, \ldots, F_{c}$ are free and $t_{i j}=\operatorname{rank}_{K}\left[H_{\mathfrak{m}}^{i}(I)\right]_{j}$.

(c) I admits an exact sequence

$$
0 \rightarrow F_{c}^{\prime} \oplus \bigoplus_{i=c}^{n} \bigoplus_{j \in \mathbb{Z}}\left(G_{i}(-j)\right)^{t_{i j}^{\prime}} \rightarrow F_{c-1}^{\prime} \rightarrow \ldots \rightarrow F_{1}^{\prime} \rightarrow I \rightarrow 0
$$

where $F_{1}^{\prime}, \ldots, F_{c}^{\prime}$ are free and $t_{i j}^{\prime}=\operatorname{rank}_{K}\left[H_{\mathfrak{m}}^{i+1-c}(I)\right]_{j}$. 
Proof. We begin with showing the equivalence of (a) and (b). Consider a $(c-1)$-presentation of the ideal $I$ :

$$
0 \rightarrow P \rightarrow E \rightarrow I \rightarrow 0
$$

It induces the following commutative diagram with exact rows:

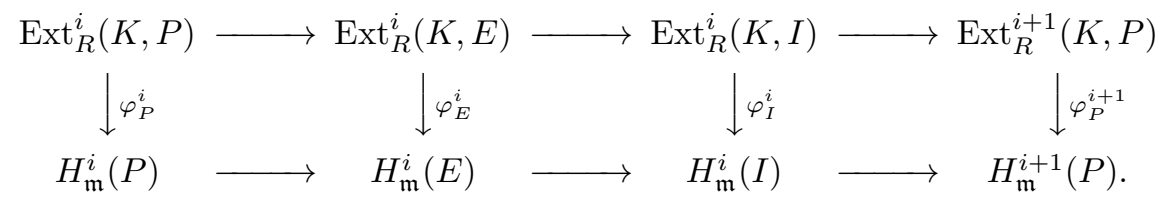

Since depth $P=n+3-c$ we see that for $i \leq n+1-c$ the map $\varphi_{E}^{i}$ is surjective if and only if $\varphi_{I}^{i}$ is surjective. The same reasoning starting with the exact sequence

$$
0 \rightarrow I \rightarrow R \rightarrow A \rightarrow 0
$$

shows that for $i<n$ the map $\varphi_{A}^{i}$ is surjective if and only if $\varphi_{I}^{i+1}$ is. It follows that $A$ is surjective-Buchsbaum if and only if $\varphi_{I}^{i}$ is surjective for all $i \leq n+1-c$. But by the definition of a $(c-1)$-presentation we have for all $i$ with $n+2-c \leq i \leq n$ that $H_{\mathfrak{m}}^{i}(E)=0$. This shows that $A$ is surjective-Buchsbaum if and only if $E$ is.

By the previous lemma $P$ has projective dimension $c-2$. Thus the assertion on the integers $t_{i j}$ follows by Proposition 4.4 and Lemma 4.3 .

In order to show the equivalence of (a) and (c) we consider the beginning of a free resolution of $X$ :

$$
0 \rightarrow N \rightarrow F_{c-1}^{\prime} \rightarrow \ldots \rightarrow F_{1}^{\prime} \rightarrow I \rightarrow 0 .
$$

According to Proposition 4.4 we have to show that $N$ is surjective-Buchsbaum of finite projective dimension if and only if $I$ has this property. This follows by shopping the exact sequence into short exact sequences and by using the following fact: Let

$$
0 \rightarrow N \rightarrow F \rightarrow M \rightarrow 0
$$

be an exact sequence of maximal modules where $F$ is free and $H_{\mathfrak{m}}^{n}(M)=0$. Then we have $H_{\mathfrak{m}}^{i}(N) \cong H_{\mathfrak{m}}^{i}(M)$ for all $i \leq n$ and $N$ is surjective-Buchsbaum of finite projective dimension if and only if $M$ has this property. This fact is proved using similar arguments as in the first part of the proof. We leave the details to the reader.

REMARK 5.3. Let $R$ be a ring which is not regular. Let $I \subset R$ be a saturated ideal which is surjective-Buchsbaum of finite projective dimension but not perfect. Let $\mathfrak{c} \subset I$ be a Gorenstein ideal with the same codimension as $I$. Then the ideal $J=\mathfrak{c}: I$ is linked to $I$ and has infinite projective dimension. Indeed, according to out last result and [26], Proposition 3.8 the ideal $J$ admits an exact sequence

$$
0 \rightarrow F_{c}^{\prime} \oplus \bigoplus_{i=1}^{n+1-c} \bigoplus_{j \in \mathbb{Z}}\left(G_{i}^{*}(+j)\right)^{t_{i j}} \rightarrow F_{c-1}^{\prime} \rightarrow \ldots \rightarrow F_{1}^{\prime} \rightarrow J(s) \rightarrow 0
$$

where $s$ is a suitable integer and the modules $F_{1}^{\prime}, \ldots, F_{c}^{\prime}$ are free. The modules $G_{i}^{*}$ are syzygy modules of the residue field $K$. Since $R$ is not regular by assumption, it follows that the $G_{i}^{*}$ have infinite projective dimension. Thus $J$ has this property too due to the exact sequence above.

Hence we have shown that the property of being surjective-Buchsbaum with finite projective dimension is not preserved in the whole liaison class of such an 
ideal $I$ if $R$ is not regular. On the other hand the property is preserved in the whole even liaison of $I$ according to [26], Theorem 3.10.

Now we want to formulate the consequences of Theorem 4.6 for an arithmetically Buchsbaum subscheme $X \subset \mathbb{P}^{n}=\mathbb{P}_{K}^{n}$. For the rest of this section we assume the field $K$ to be infinite. We denote by $I(X) \subset R$ the homogeneous ideal of $X$. Then $A=R / I(X)$ is the homogeneous coordinate ring of $X$. Recall that a subscheme $X$ is said to be arithmetically Buchsbaum if its homogeneous coordinate ring is Buchsbaum. As usual we denote the ideal sheaf of $X$ by $\mathcal{J}_{X}$.

Then we have.

Corollary 5.4. Let $X \subset \mathbb{P}^{n}$ be a subscheme of codimension c. Then the following conditions are equivalent:

(a) $X$ is arithmetically Buchsbaum.

(b) $\mathfrak{m} \cdot H_{*}^{i}\left(\mathcal{J}_{X \cap L}\right)=0$ for any linear subspace $L \subset \mathbb{P}^{n}$ of dimension $>c$ intersecting $X$ transversally and all $i$ with $1 \leq i \leq \operatorname{dim} L-c$.

(c) There is an exact sequence

$$
0 \rightarrow \mathcal{F}_{c} \rightarrow \ldots \rightarrow \mathcal{F}_{2} \rightarrow \mathcal{F}_{1} \oplus \bigoplus_{j}\left(\Omega_{\mathbb{P}^{n}}^{p_{j}}\left(-e_{j}\right)\right)^{s_{j}} \rightarrow \mathcal{J}_{X} \rightarrow 0
$$

where $\mathcal{F}_{1}, \ldots, \mathcal{F}_{c}$ are direct sums of line bundles and $1 \leq p_{j} \leq n-c$.

(d) There is an exact sequence

$$
0 \rightarrow \mathcal{F}_{c}^{\prime} \oplus \bigoplus_{j}\left(\Omega_{\mathbb{P}^{n}}^{p_{j}^{\prime}}\left(-e_{j}^{\prime}\right)\right)^{s_{j}^{\prime}} \rightarrow \mathcal{F}_{c-1}^{\prime} \rightarrow \ldots \rightarrow \mathcal{F}_{1}^{\prime} \rightarrow \mathcal{J}_{X} \rightarrow 0
$$

where $\mathcal{F}_{1}^{\prime}, \ldots, \mathcal{F}_{c}^{\prime}$ are direct sums of line bundles and $c \leq p_{j}^{\prime} \leq n-1$.

Proof. The equivalence of (a) and (b) is shown in 33 . Hence the claim follows by Theorem 5.2 using $H_{*}^{i}\left(\mathcal{J}_{X}\right) \cong H_{\mathfrak{m}}^{i+1}(I(X))$ if $i \geq 1$ and $\tilde{G}_{i} \cong \Omega_{\mathbb{P n}}^{i-1}$ by Lemma 4.3 .

REMARK 5.5. (i) The last statement is a generalization of the main result of Chang in [6]. She proved it for subschemes of codimension 2. The result has been proved independently by C. Walter (unpublished).

(ii) Since $\left(\Omega_{\mathbb{p} n}^{p}\right)^{*} \cong \Omega_{\mathbb{p} n}^{n-p}(n+1)$ the corollary shows in particular that any arithmetically Buchsbaum subscheme of codimension $c$ is the zero scheme of a section of a vector bundle $\mathcal{E}=\oplus_{j}\left(\Omega_{\mathbb{p} n}^{q_{j}}\left(f_{j}\right)\right)^{s_{j}}$ with $c \leq q_{j} \leq n$. This section cannot be a general one if $\mathcal{E}$ is not a direct sum of line bundles because then $\mathcal{E}$ has rank $\geq n$.

Following Chang we want to introduce a name for the exact sequence described in part (c) of the corollary above.

Definition 5.6. A subscheme $X$ of codimension $c$ is said to have an $\Omega$-resolution if there exists an exact sequence

$$
0 \rightarrow \mathcal{F}_{c} \stackrel{\alpha_{c}}{\longrightarrow} \ldots \rightarrow \mathcal{F}_{2} \stackrel{\alpha_{2}}{\longrightarrow} \mathcal{F}_{1} \oplus \bigoplus_{j}\left(\Omega_{\mathbb{P}^{n}}^{p_{j}}\left(-e_{j}\right)\right)^{s_{j}} \rightarrow \mathcal{J}_{X} \rightarrow 0
$$

where $\mathcal{F}_{1}, \ldots, \mathcal{F}_{c}$ are finite direct sums of line bundles, $1 \leq p_{j} \leq n-c, 1 \leq s_{j}$ and $\left(p_{j}, e_{j}\right)$ are all distinct ordered pairs of integers.

The $\Omega$-resolution is said to be minimal if there is no line bundle $\mathcal{L}$ in the resolution such that the restriction of $\alpha_{i}(2 \leq i \leq c)$ to $\mathcal{L}$ induces an isomorphism of $\mathcal{L}$ onto $\mathcal{L}$. 
Corollary 5.4 says that a subscheme $X$ has an $\Omega$-resolution if and only if it is arithmetically Buchsbaum.

It is not difficult to see that the numbers $p_{j}, e_{j}, s_{j}$ in the $\Omega$-resolution are uniquely determined by $X$ because $h^{p_{j}}\left(\mathcal{J}_{X}\left(e_{j}\right)\right)=s_{j}$ are the only non-zero intermediate cohomology groups of $\mathcal{J}_{X}$. In fact, there is a stronger uniqueness property which shows that an $\Omega$-resolution is indeed a locally free resolution in the sense of the introduction.

LEMmA 5.7. The minimal $\Omega$-resolution of an arithmetically Buchsbaum subscheme is uniquely determined (up to isomorphism).

Proof. Every minimal $\Omega$-resolution of $X$ gives rise to a minimal $(c-1)$ presentation $0 \rightarrow P \rightarrow E \rightarrow I(X) \rightarrow 0$ and a minimal free resolution of $P$ and vice versa. But a minimal $(c-1)$-presentation is uniquely determined due to Theorem 3.4.

Remark 5.8. Putting together Corollary 5.4 and Lemma 5.7 we obtain Theorem 1.2 of the introduction.

Now we want to draw some consequences of the existence of a $\Omega$-resolution. Since the Koszul complex provides a minimal free resolution of $\Omega_{\mathbb{P} n}^{p}$ the mapping cone construction implies the following information on the free resolution of $X$.

Corollary 5.9. If $X$ has an $\Omega$-resolution as in Definition 5.8 then $\mathcal{J}_{X}$ has a (possibly non-minimal) free resolution of the form:

$$
\begin{aligned}
0 \rightarrow & \bigoplus_{p_{j}=1}\left(\mathcal{O}\left(-e_{j}-n-1\right)\right)^{s_{j}} \rightarrow \bigoplus_{1 \leq p_{j} \leq 2}\left(\mathcal{O}\left(-e_{j}-p_{j}-n+1\right)\right)^{s_{j}\left(\begin{array}{c}
n+1 \\
p_{j}+n-1
\end{array}\right)} \rightarrow \ldots \\
& \rightarrow \bigoplus_{1 \leq p_{j} \leq n-c}\left(\mathcal{O}\left(-e_{j}-p_{j}-c-1\right)\right)^{s_{j}\left(\begin{array}{c}
n+1 \\
p_{j}+c+1
\end{array}\right)} \\
& \rightarrow \mathcal{F}_{c} \oplus \bigoplus_{j}\left(\mathcal{O}\left(-e_{j}-p_{j}-c\right)\right)^{s_{j}\left(\begin{array}{c}
n+1 \\
p_{j}+c
\end{array}\right)} \\
& \rightarrow \ldots \rightarrow \mathcal{F}_{1} \oplus \bigoplus_{j}\left(\mathcal{O}\left(-e_{j}-p_{j}-1\right)\right)^{s_{j}\left(\begin{array}{c}
n+1 \\
p_{j}+1
\end{array}\right)} \rightarrow \mathcal{J}_{X} \rightarrow 0 .
\end{aligned}
$$

Remark 5.10. Let $t$ be the depth of $A=R / I(X)$. Suppose $t<\operatorname{dim} A$. Then a slight generalization of a result of Rao [29], Theorem 2.5 implies that the last free module in the minimal free resolution of $A$ equals the last free module in the minimal free resolution of $H_{\mathfrak{m}}^{t}(A)$ if the latter has finite length. In case $A$ is Buchsbaum a lot more is true. The previous corollary implies that the last $n+1-t-c$ free modules in the minimal free resolution of $\mathcal{J}_{X}$ are completely determined by the intermediate cohomologies of $X$ because at these spots cancellation is impossible. This means using the previous notation

$$
\operatorname{Tor}_{i}^{R}(K, A) \cong \bigoplus_{j}\left(K\left(-e_{j}-p_{j}-i\right)\right)^{s_{j}\left(\begin{array}{c}
n+1 \\
p_{j}+i
\end{array}\right)} \quad \text { if } i \geq c+1 .
$$

Next, we want to describe the $\Omega$-resolution of the general hyperplane section of a subscheme having an $\Omega$-resolution. 
Lemma 5.11. Let $X$ be a subscheme having an $\Omega$-resolution as in Definition 5.6. Let $H \subset \mathbb{P}^{n}$ be a general hyperplane. Then $X \cap H$ has an $\Omega$-resolution as follows:

$$
\begin{aligned}
&\left.0 \rightarrow \mathcal{F}_{c}\right|_{H} \oplus \bigoplus_{p_{j}=n-c}\left(\mathcal{O}_{H}\left(-e_{j}-n\right)\right)^{s_{j}\left(\begin{array}{l}
n \\
0
\end{array}\right)} \rightarrow \ldots\left.\rightarrow \mathcal{F}_{2}\right|_{H} \oplus \bigoplus_{p_{j}=n-c}\left(\mathcal{O}_{H}\left(-e_{j}-n-2+c\right)\right)^{s_{j}\left(\begin{array}{c}
n \\
c-2
\end{array}\right)} \\
&\left.\rightarrow \mathcal{F}_{1}\right|_{H} \oplus \bigoplus_{p_{j}=1}\left(\mathcal{O}_{H}\left(-e_{j}-1\right)\right)^{s_{j}} \oplus \bigoplus_{p_{j}=n-c}\left(\mathcal{O}_{H}\left(-e_{j}-n-1+c\right)\right)^{s_{j}\left(\begin{array}{c}
n \\
c-1
\end{array}\right) \oplus} \\
& \bigoplus_{1 \leq p_{j}<n-c}\left(\Omega_{H}^{p_{j}}\left(-e_{j}\right)\right)^{s_{j}} \oplus \bigoplus_{2 \leq p_{j} \leq n-c}\left(\Omega_{H}^{p_{j}-1}\left(-e_{j}-1\right)\right)^{s_{j}} \\
& \rightarrow \mathcal{J}_{X \cap H} \rightarrow 0 .
\end{aligned}
$$

Proof. We will follow the approach described in Lemma 3.6. It is well-known that

$$
\left.\Omega_{\mathbb{P n}}^{p}\right|_{H} \cong \Omega_{H}^{p} \oplus \Omega_{H}^{p-1}(-1) .
$$

Write the second term in the given $\Omega$-resolution of $X$ as $\mathcal{E} \oplus \bigoplus_{p_{j}=n-c}\left(\Omega_{\mathbb{P}^{n}}^{n-c}\left(-e_{j}\right)\right)^{s_{j}}$. Then its restriction to $H$ can be obtained by applying the last formula. We have to find a $(c-1)$-presentation of this restriction. Fortunately this is easy. We obtain by resolving $\bigoplus_{p_{j}=n-c}\left(\Omega_{H}^{n-c}\left(-e_{j}\right)\right)^{s_{j}}$ the $(c-1)$-presentation

$$
\begin{aligned}
0 \rightarrow & \bigoplus_{p_{j}=n-c}\left(\Omega_{H}^{n-c+1}\left(-e_{j}\right)\right)^{s_{j}} \rightarrow \\
& \left.\mathcal{E}\right|_{H} \oplus \bigoplus_{p_{j}=n-c}\left(\Omega_{H}^{n-c-1}\left(-e_{j}-1\right)\right)^{s_{j}} \oplus \bigoplus_{p_{j}=n-c}\left(\mathcal{O}_{H}\left(-e_{j}-n-1+c\right)\right)^{s_{j}\left(\begin{array}{c}
n \\
c-1
\end{array}\right)} \rightarrow \\
& \left.\mathcal{E}\right|_{H} \oplus \bigoplus_{p_{j}=n-c}\left(\Omega_{H}^{n-c-1}\left(-e_{j}-1\right)\right)^{s_{j}} \oplus \bigoplus_{p_{j}=n-c}\left(\Omega_{H}^{n-c}\left(-e_{j}\right)\right)^{s_{j}} \rightarrow 0 .
\end{aligned}
$$

The Koszul complex provides a resolution of the term on the left-hand side. Thus an application of the Horseshoe lemma gives the desired $\Omega$-resolution as in Lemma 3.6.

Roughly speaking, we get the $\Omega$-resolution of the hyperplane section by restricting any bundle occurring in the $\Omega$-resolution of $X$ to $H$ and then replacing $\bigoplus_{p_{j}=n-c}\left(\Omega_{H}^{n-c}\left(-e_{j}\right)\right)^{s_{j}}$ by its free resolution.

Cutting $X$ by $n-c$ general hyperplanes we obtain a zero-dimensional subscheme $Z$. Repeated use of the lemma above provides an $\Omega$-resolution of $Z$ which is in fact a free resolution reflecting the fact that $Z$ is arithmetically Cohen-Macaulay.

Next, we want to consider abelian varieties. We need the following sufficient but not necessary Buchsbaum criteria (cf., for example, [33], Proposition I.3.10) which generalizes Lemma 1.2 .

LEMmA 5.12. Let $X \subset \mathbb{P}^{n}$ be a subscheme such that the following two conditions are satisfied:

(i) $\mathfrak{m} \cdot H_{*}^{i}\left(\mathcal{J}_{X}\right)=0$ for all $i$ with $1 \leq i \leq \operatorname{dim} X$.

(ii) If $H^{i}\left(\mathcal{J}_{X}(e)\right) \neq 0$ and $H^{j}\left(\mathcal{J}_{X}(f)\right) \neq 0$ for some integers $i, j$ with $1 \leq i<$ $j \leq \operatorname{dim} X$ then $(i+e)-(j+f) \neq 1$. 
Then $X$ is arithmetically Buchsbaum.

EXAMPLE 5.13. Let $\mathcal{L}$ be an ample line bundle on an abelian variety $Y$ of dimension $g$. Then $\mathcal{L}^{m}$ is very ample if $m \geq 3$ according to a result of Lefschetz (cf. 22], p. 163). In this case $\mathcal{L}^{m}$ provides an embedding of $Y$ into $\mathbb{P}\left(H^{0}\left(Y, \mathcal{L}^{m}\right)\right)$. Let us denote the image by $X$. Then $X$ is projectively normal due to Koizumi and Ohbuchi (cf. 20], Theorem 7.3.1) and its homogeneous ideal is generated by quadrics if $m \geq 4$ according to Kempf [19]. From the results on the cohomology of line bundles on an abelian variety [22, Section 16 it follows that

$$
H_{*}^{i}\left(\mathcal{J}_{X}\right) \cong K^{(\stackrel{g}{i-1})} \quad \text { if } 2 \leq i \leq g .
$$

Hence if $m \geq 4$ then $X$ is arithmetically Buchsbaum by the lemma above and has an $\Omega$-resolution of the form:

$$
0 \rightarrow \mathcal{F}_{c} \rightarrow \ldots \rightarrow \mathcal{F}_{2} \rightarrow \mathcal{O}_{\mathbb{P}^{n}}^{\alpha}(-2) \oplus \bigoplus_{2 \leq i \leq g}\left(\Omega_{\mathbb{P}^{n}}^{i}\right)^{\left({ }_{i-1}\right)} \rightarrow \mathcal{J}_{X} \rightarrow 0
$$

where $c=h^{0}\left(Y, \mathcal{L}^{m}\right)-1-g$ and $\alpha>0$. Using the fact that $X$ is subcanonical this resolution can be described a little more detailed. It follows for example that $\mathcal{F}_{c}=\mathcal{O}(-g-c)$ if the $\Omega$-resolution above is minimal.

In 28] Pareschi has obtained information on the beginning of the minimal free resolution of $X$ by showing that it is linear in the first stages. The $\Omega$-resolution of $X$ above allows to determine the end of the minimal free resolution of $X$ (cf. Remark 5.10). It follows immediately that the whole free resolution of $X$ cannot be linear.

Finally we want to collect some information on the twists of the line bundles occurring in a minimal $\Omega$-resolution. We will use the following notation for a graded $R$-module $N$ :

and

$$
e(N)=\sup \left\{j \in \mathbb{Z} \mid[N]_{j} \neq 0\right\}
$$

$$
e^{+}(N)=e(N / \mathfrak{m} N)
$$

Note that $e^{+}(N)$ is just the maximal degree of a minimal generator of $N$ if $N$ is finitely generated.

Proposition 5.14. Suppose $X$ has a minimal $\Omega$-resolution as in Definition 5.0. Let

$$
e(X)=e\left(H_{*}^{n-c+1}\left(\mathcal{J}_{X}\right)\right)
$$

be the index of speciality and let $\mathcal{F}_{i}=\oplus_{k} \mathcal{O}_{\mathbb{P}^{n}}\left(-d_{i k}\right)(1 \leq i \leq c)$. Then it holds:

$$
1 \leq p_{j}+e_{j} \leq e(X)+n+2-c \text { for all } j
$$

$$
\min _{j, k}\left\{d_{1 k}, p_{j}+e_{j}\right\}-1 \leq d_{i k}-i \leq e(X)+n+1-c \quad \text { if } 1 \leq i \leq c .
$$

Proof. Taking global sections the given $\Omega$-resolution provides a minimal $(c-$ 1)-presentation of $I=I(X)$ :

$$
0 \rightarrow P \rightarrow E \rightarrow I \rightarrow 0
$$

and a minimal free resolution of $P$ :

$$
0 \rightarrow F_{c} \rightarrow \ldots \rightarrow F_{2} \rightarrow P \rightarrow 0
$$


First let us suppose $c \geq 3$. Since $\operatorname{Ext}_{R}^{i}(P, R)=0$ if $i>0$ and $i \neq c-2$ dualizing the resolution of $P$ with respect to $R$ gives the exact sequence:

$$
0 \rightarrow P^{*} \rightarrow F_{2}^{*} \rightarrow \ldots \rightarrow F_{c}^{*} \rightarrow \operatorname{Ext}_{R}^{c-2}(P, R) \rightarrow 0 .
$$

Moreover, we get the exact sequence

$$
0 \rightarrow R \rightarrow E^{*} \rightarrow P^{*} \rightarrow 0 .
$$

Let $y \in E^{*}$ be the image of the unit element of $R$. Then $I$ is the order ideal of $y$. Since $E^{*}$ is a $(c+1)$-syzygy by the explicit description of $E$ (cf. Corollary 5.4) and $I$ has codimension $c$ it follows by $\mathbf{1 0}$, Theorem 3.14 that $y$ is not a minimal generator of $E^{*}$. This implies in particular that $a\left(E^{*}\right)=a\left(P^{*}\right)$ and $e^{+}\left(E^{*}\right)=e^{+}\left(P^{*}\right)$. Now we have

$$
(\tilde{E})^{*} \cong \mathcal{F}_{1}^{*} \oplus \bigoplus_{j}\left(\Omega_{\mathbb{P}^{n}}^{n-p_{j}}\left(n+1+e_{j}\right)\right)^{s_{j}}
$$

It follows

$(* *) \quad a\left(E^{*}\right)=\min _{j, k}\left\{-d_{1 k},-p_{j}-e_{j}\right\} \quad$ and $\quad e^{+}\left(E^{*}\right)=\max _{j, k}\left\{-d_{1 k},-p_{j}-e_{j}\right\}$.

According to Lemma 5.1 $P^{*}$ is a minimal $(c-1)$-syzygy of $K_{A}(n+1) \cong \operatorname{Ext}_{R}^{c-2}(P, R)$. Thus we obtain

$$
a\left(E^{*}\right)=a\left(P^{*}\right) \geq a\left(K_{A}(n+1)\right)+c-1=-e(X)-n-1+c-1 .
$$

Hence $(* *)$ provides

$$
\max _{j, k}\left\{d_{1 k}, p_{j}+e_{j}\right\} \leq e(X)+n+2-c .
$$

This proves the estimate on the right-hand side of claim (a) and also of claim (b) taking $(*)$ into account again.

Since $P$ does not split a free direct summand by Lemma 5.1 we obtain using Theorem I.4.1 of 25 that

$$
\min _{j, k}\left\{d_{2 k}\right\}=a(P) \geq 1-e^{+}\left(P^{*}\right)=1-e^{+}(E *) .
$$

Hence $(* *)$ implies the estimate on the left-hand side in claim (b).

It remains to show the estimate on the left-hand side in claim (a). Let $C$ be the curve arising as intersection of $X$ with a general linear subspace of dimension $c+1$. Then we get using for example Lemma 5.11 that

$$
H_{*}^{1}\left(\mathcal{J}_{C}\right) \cong \bigoplus_{i=0}^{n-1-c}\left(H_{*}^{1+i}\left(\mathcal{J}_{X}\right)(-i)\right)^{\left(\begin{array}{c}
n-c-1 \\
i
\end{array}\right)}
$$

It follows

$$
a\left(H_{\mathfrak{m}}^{1}\left(\mathcal{J}_{C}\right)\right)=\min \left\{a\left(H_{*}^{1+i}\left(\mathcal{J}_{X}\right)\right)+i\right\}=\min \left\{e_{j}+p_{j}\right\}-1 .
$$

Let $H$ be a general hyperplane and consider the exact sequence

$$
H_{*}^{0}\left(\mathcal{J}_{C \cap H}\right) \rightarrow H_{*}^{1}\left(\mathcal{J}_{C}\right)(-1) \stackrel{H}{\longrightarrow} H_{*}^{1}\left(\mathcal{J}_{C}\right) .
$$

Since $C$ is arithmetically Buchsbaum the map on the right-hand side is zero. Thus we obtain

$$
a\left(H_{*}^{1}\left(\mathcal{J}_{C}\right)\right) \geq 0
$$

completing the proof in case $c \geq 3$. 
Let now $c=2$. Then the claims follow similarly using the exact sequence

$$
0 \rightarrow R \rightarrow E^{*} \rightarrow P^{*} \rightarrow \operatorname{Ext}_{R}^{1}(I, R) \rightarrow 0 .
$$

REMARK 5.15. In case codim $X=2$ there is a considerably stronger result. In fact, Chang [6], Theorem 2.3 could characterize the possible twists in an $\Omega$ resolution precisely if $c=2$. (Her results are also true in positive characteristic due to Walter 35.) However, it seems to be rather difficult to achieve a similarly precise characterization in higher codimension. The corresponding problem is even open for arithmetically Cohen-Macaulay subschemes.

ExAmPle 5.16. Let $n \geq 3$ and $r \geq n-2$ be two integers. There is an arithmetically Buchsbaum subscheme $X$ with $\Omega$-resolution

$$
0 \rightarrow\left(\mathcal{O}_{\mathbb{P}^{n}}(-r-1)\right)^{n-2} \oplus \mathcal{O}_{\mathbb{P}^{n}}(n-2 r-3) \rightarrow \Omega_{\mathbb{P}^{n}}(-r+1) \rightarrow \mathcal{J}_{X} \rightarrow 0
$$

where $e(X)=2(r-n+1)$ (cf. [7], Lemma 6.10). For $X$ both estimates in Proposition 5.14(b) are optimal whereas the estimate on the right-hand side in Proposition 5.14(a) is attained if $r=n-2$ and the one on the left-hand side is optimal too if $r=n-2=1$.

REMARK 5.17. It has been shown as Corollary 5.4 in 26 that an arithmetically Buchsbaum scheme $X \subset \mathbb{P}^{n}$ is minimal in its even liaison class if the estimate on the right-hand side in Proposition 5.14(a) is attained. Fixing any codimension $c \geq 2$ various such schemes do exist (cf. [26], Example 8.4).

We conclude this section by pointing out that the previous result implies 15, Corollary 2.8. Recall that the Castelnuovo-Mumford regularity of a subscheme $X$ (cf. [21]) is the integer

$$
\operatorname{reg} X=\max \left\{i+1+e\left(H_{*}^{i}\left(\mathcal{J}_{X}\right) \mid i \geq 1\right\} .\right.
$$

Corollary 5.18. If $X$ is arithmetically Buchsbaum of codimension $c$ then

$$
e(X)+n+2-c \leq \operatorname{reg} X \leq e(X)+n+3-c .
$$

Proof. By definition of the regularity we have

$$
\operatorname{reg} X-1=\max \left\{i+e\left(H_{*}^{i}\left(\mathcal{J}_{X}\right)\right) \mid i>0\right\} \geq e(X)+n+1-c .
$$

Using the notation of the previous proposition we also observe that

$$
\max \left\{i+e\left(H_{*}^{i}\left(\mathcal{J}_{X}\right)\right) \mid 1 \leq i \leq n-c\right\}=\max \left\{e_{j}+p_{j}\right\} .
$$

Thus the assertion follows by Proposition 5.14 (a).

In general it is not easy to compute the index of speciality. In [24, Lemma 4.6 the estimate

$$
e(X) \leq \operatorname{deg} X-\operatorname{dim} X-2
$$

is shown. If $X$ is an integral subscheme there is the better estimate (cf. 24, Lemma 4.6)

$$
e(X)<\left\lceil\frac{\operatorname{deg} X-1}{c}\right\rceil-\operatorname{dim} X
$$


REMARK 5.19. (i) We have left open the rather difficult smoothness and integrality questions. However, there are useful criteria for arithmetically Buchsbaum subschemes of codimension two due to Chang [6], Theorem 2.2 and 2.3. The smooth integral arithmetically Buchsbaum subschemes of arbitrary codimension which are divisors on a variety of minimal degree have been described in [27].

(ii) A different approach to study an arithmetically Buchsbaum subscheme $X$ of $\mathbb{P}^{n}$ has been proposed by M. Amasaki. Using the degrees of the elements of a Gröbner basis of $I(X)$ in generic coordinates he obtains a rough classification of arithmetically Buchsbaum subschemes of projective space (cf. [1] Theorem 4.5 and Corollary 6.7).

(iii) Notice that our structural approach allows to consider not only subschemes of $\mathbb{P}^{n}$ but even subschemes of some arithmetically Gorenstein subscheme $G$. Indeed, our characterization of arithmetically Buchsbaum subschemes of $\mathbb{P}^{n}$ has been obtained by specializing the results about surjective-Buchsbaum subschemes of $G$.

\section{Quasi-Buchsbaum subschemes}

In this section we show that every ideal of a Gorenstein ring admits an exact sequence where all the occurring modules except the ideal itself are EilenbergMacLane modules. As a consequence we obtain that every equidimensional CohenMacaulay subscheme of projective space admits a locally free resolution consisting of Eilenberg-MacLane bundles. From this result we derive our characterization of quasi-Buchsbaum subschemes by means of weak $\Omega$-resolutions.

The construction in the proof of the following statement works for arbitrary modules. However, for simplicity and keeping our applications in mind we restrict ourselves to saturated ideals.

Theorem 6.1. Let $I \subset R$ be a saturated ideal of codimension c. Put $A=R / I$ and

$$
s=\min \left\{k \geq c \mid H_{\mathfrak{m}}^{j}(A)=0 \text { for all } j \text { with } k+1 \leq j \leq n-k\right\} .
$$

Then $I$ admits an exact sequence

$$
0 \rightarrow E_{s} \rightarrow E_{s-1} \rightarrow \ldots \rightarrow E_{1} \rightarrow I \rightarrow 0
$$

where all the modules $E_{i}$ are Eilenberg-MacLane modules of depth $2 i$ or maximal Cohen-Macaulay modules, the module $E_{i}$ has finite finite projective dimension if $2 \leq i \leq s$ and $E_{1}$ has finite finite projective dimension if and only if I does.

Furthermore, the $R$-module $E_{i}$ is free if $i>n-c$ and we have

$$
H_{\mathfrak{m}}^{2 i}\left(E_{i}\right) \cong H_{\mathfrak{m}}^{i}(A) \quad \text { if } i \leq \min \{n-c, s\} .
$$

Moreover, the sequence $(+)$ is uniquely determined up to isomorphisms of exact sequences if it is impossible to cancel out free direct summands. In this case (+) is called minimal.

If $s=n-c$ then all the modules $E_{i}$ have cohomology of finite length if and only if $A$ is equidimensional and locally Cohen-Macaulay.

Proof. First we observe that the integer $s$ is well-defined. Indeed, the condition in its definition is trivially satisfied if $k \geq \frac{n}{2}$. Thus we have $s=c$ if $c \geq \frac{n}{2}$ and $c \leq s \leq \frac{n}{2}$ otherwise. Let $d=n+1-c$ denote the dimension of $R / I$. Now we proceed in several steps. 
(I) We show that for any integer $k$ where $2 \leq k \leq \min \{d, c\}$ there is an exact sequence

$$
0 \rightarrow P_{k} \rightarrow E_{k-1} \rightarrow \ldots \rightarrow E_{1} \rightarrow I \rightarrow 0
$$

where the modules $E_{i}$ have the properties as claimed in our assertion and $P_{k}$ is a module with finite projective dimension and

$$
H_{\mathfrak{m}}^{j}\left(P_{k}\right) \cong \begin{cases}0 & \text { if } j<2 k \\ H_{\mathfrak{m}}^{j-k}(A) & \text { if } 2 k \leq j \leq n .\end{cases}
$$

In order to prove this we induct on $k \geq 2$. If $k=2$ we take as $\left(C_{2}\right)$ a minimal $(n-2)$-presentation of $I$. It has the desired properties due to Lemma 3.2. Now let $2<k \leq \min \{d, c\}$. By induction we have an exact sequence

$\left(C_{k-1}\right) \quad 0 \rightarrow P_{k-1} \rightarrow E_{k-2} \rightarrow \ldots \rightarrow E_{1} \rightarrow I \rightarrow 0$

where $H_{\mathfrak{m}}^{d+k-1}\left(P_{k-1}\right) \cong H_{\mathfrak{m}}^{d}(A) \neq 0$ because $2 k-2<d+k-1<d+c=n+1$. Consider a minimal $(n+2-2 k)$-presentation

$$
0 \rightarrow P_{k} \rightarrow E_{k-1} \rightarrow P_{k-1} \rightarrow 0
$$

of the module $P_{k-1}$. Then we obtain by induction and Lemma 3.2

$$
H_{\mathfrak{m}}^{j}\left(E_{k-1}\right) \cong\left\{\begin{array} { l l } 
{ H _ { \mathfrak { m } } ^ { j } ( P _ { k - 1 } ) } & { \text { if } j < 2 k - 1 } \\
{ 0 } & { \text { if } 2 k - 1 \leq j \leq n }
\end{array} \cong \left\{\begin{array}{ll}
H_{\mathfrak{m}}^{k-1}(A) & \text { if } j=2 k-2 \\
0 & \text { if } j \neq 2 k-2, n+1
\end{array}\right.\right.
$$

and

$$
H_{\mathfrak{m}}^{j}\left(P_{k}\right) \cong\left\{\begin{array} { l l } 
{ 0 } & { \text { if } j \leq 2 k - 1 } \\
{ H _ { \mathfrak { m } } ^ { j - 1 } ( P _ { k - 1 } ) } & { \text { if } 2 k \leq j \leq n }
\end{array} \cong \left\{\begin{array}{ll}
0 & \text { if } j<2 k \\
H_{\mathfrak{m}}^{j-k}(A) & \text { if } 2 k \leq j \leq n .
\end{array}\right.\right.
$$

In particular, because of $2 k-2<d+k-1 \leq n$ we see that $H_{\mathfrak{m}}^{d+k-1}\left(E_{k-1}\right)=0$ is not isomorphic to $H_{\mathfrak{m}}^{d+k-1}\left(P_{k-1}\right) \neq 0$. Thus, $P_{k}$ cannot be trivial. Therefore splicing together the sequence $\left(C_{k-1}\right)$ and the minimal $(n+2-2 k)$-presentation of $P_{k-1}$ yields the desired exact sequence $\left(C_{k}\right)$.

(II) Now we distinguish two cases.

Case 1: Suppose that $d<c$. Thus we get $s=c$. Let $d \geq 2$. Then we have an exact sequence $\left(C_{d}\right)$ by step (I) of the proof. The module $P_{d}$ has finite projective dimension and satisfies

$$
H_{\mathfrak{m}}^{j}\left(P_{d}\right) \cong \begin{cases}0 & \text { if } j \leq 2 d-1 \\ H_{\mathfrak{m}}^{j-d}(A) & \text { if } 2 d \leq j \leq n .\end{cases}
$$

Thus $P_{d}$ has depth $2 d$ since $2 d \leq n$ due to our assumption of this case. It follows by the Auslander-Buchsbaum formula that $P_{d}$ has a minimal free resolution

$$
0 \rightarrow F_{n+1-2 d} \rightarrow \ldots \rightarrow F_{0} \rightarrow P_{d} \rightarrow 0 .
$$

Splicing together this resolution and the sequence $\left(C_{d}\right)$ yields the desired sequence $(+)$ of length $s=c$ where we relabel the modules $F_{i}$ as $E_{d+i}$.

If $d=1$ then we consider a minimal $(n-1)$-presentation of $I$

$$
0 \rightarrow P \rightarrow E_{1} \rightarrow I \rightarrow 0 .
$$

Since $I$ is saturated we obtain by Lemma 3.2 that $E_{1}$ is a maximal Cohen-Macaulay module and that $P$ is an Eilenberg-MacLane module of depth 3. Thus, replacing $P$ by its minimal free resolution provides the desired sequence $(+)$ of length $s=n$. Case 2: Suppose that $d \geq c$. Thus we have an exact sequence $\left(C_{c}\right)$ by step (I). We 
claim that $I$ admits even an exact sequence $\left(C_{s}\right)$ with the properties described in step (I). Indeed, continuing in the fashion of step (I) we just need to check that the modules $P_{c}, \ldots, P_{s}$ are non-trivial. If $t=c$ we are done by step (I). If $c<k \leq s$ and we have constructed $P_{k-1}$ we know by the definition of $s$ that there is an integer $j$ such that $2 k-1 \leq j \leq n$ and $H_{\mathfrak{m}}^{j}\left(P_{k-1}\right) \cong H_{\mathfrak{m}}^{j-k+1}(A) \neq 0$. Since $H_{\mathfrak{m}}^{j}\left(E_{k-1}\right)=0$ if $2 k-1 \leq j \leq n$ it follows that the module $P_{k}$ cannot be trivial by considering the minimal $(n+2-2 k)$-presentation

$$
0 \rightarrow P_{k} \rightarrow E_{k-1} \rightarrow P_{k-1} \rightarrow 0 .
$$

Now we turn our attention to the module $P_{s}$ occurring in $\left(C_{s}\right)$. It has depth $\geq 2 s$ by construction. Furthermore, we have that $H_{\mathfrak{m}}^{j}\left(P_{s}\right) \cong H_{\mathfrak{m}}^{j-s}(A) \neq 0$ if $2 s+1 \leq j \leq n$ due to the definition of $s$. Therefore $P_{s}$ is an Eilenberg-MacLane module. Thus, defining $E_{s}=P_{s}$ the sequence $\left(C_{s}\right)$ gives the desired sequence $(+)$.

(III) Given a minimal sequence (+) we can reverse the constructions in steps (I) and (II) by shopping it into shorter exact sequences. Thus the claimed uniqueness is a consequence of the uniqueness properties of $q$-presentations and free resolutions.

The final claim follows because in case $s \geq n-c$ all the modules $E_{i}$ in $(+)$ have cohomology of finite length if and only if $A$ has cohomology of finite length. The latter is true if and only if $A$ is equidimensional and locally Cohen-Macaulay.

REMARK 6.2. Using the notation of the proposition above let us assume that $I$ is a perfect ideal. Then we obtain $s=c$ and the minimal sequence $(+)$ is nothing else than the minimal free resolution of $I$.

Observe that the sequence $(+)$ above can have a length which is less than the dimension of $A$. This is also reflected in the following result where we use the modules $G_{i}$ defined immediately before Lemma 4.3 .

COROLlary 6.3. Let $I \subset R$ be a saturated ideal of finite projective dimension. Put $s=\min \left\{k \geq c \mid H_{\mathfrak{m}}^{j}(R / I)=0\right.$ for all $j$ with $\left.k+1 \leq j \leq n-k\right\}$ and $v=\min \{n-c, s\}$. Then the following conditions are equivalent:

(a) It holds

$$
\mathfrak{m} \cdot H_{\mathfrak{m}}^{i}(R / I)=0 \quad \text { if } 1 \leq i \leq v .
$$

(b) I admits an exact sequence

$$
\begin{aligned}
0 \rightarrow F_{s} \rightarrow \ldots \rightarrow F_{v+1} \rightarrow F_{v} \oplus \bigoplus_{j}\left(G_{2 v}\left(-e_{v . j}\right)\right)^{s_{v . j}} & \rightarrow \ldots \\
& \rightarrow F_{1} \oplus \bigoplus_{j}\left(G_{2}\left(-e_{1 . j}\right)\right)^{s_{1 . j}} \rightarrow I \rightarrow 0
\end{aligned}
$$

where the integers $s_{i . j}$ are non-negative.

Proof. Consider the exact sequence $(+)$ given by Theorem 6.1. If (a) is satisfied then the modules $E_{i}$ are free if $v<i \leq s$ and are Eilenberg-MacLane modules of finite projective dimension where $\mathfrak{m} \cdot H_{\mathfrak{m}}^{2 i}\left(E_{i}\right)=0$ otherwise. Hence the $E_{i}, 1 \leq i \leq v$, are surjective-Buchsbaum modules by Lemma 4.2. Therefore Proposition 4.6 shows $E_{i} \cong F_{i} \oplus \bigoplus_{j}\left(G_{2 i}\left(-e_{i . j}\right)\right)^{s_{i . j}}$ for some free module $F_{i}$. Thus the sequence $(+)$ is of the form as claimed in (b). 
In order to show the converse we use the sequence in (b) to compute

$$
H_{\mathfrak{m}}^{i}(R / I) \cong H_{\mathfrak{m}}^{2 i}\left(G_{2 i}\right) \quad \text { if } 1 \leq i \leq v
$$

Since the modules $G_{i}$ are Buchsbaum we obtain (a).

Now we specialize our results to the case where $R$ is a polynomial ring.

Corollary 6.4. Let $X \subset \mathbb{P}^{n}$ be an equidimensional Cohen-Macaulay subscheme of codimension $c$. Then $X$ admits a locally free resolution

$$
0 \rightarrow \mathcal{E}_{s} \rightarrow \ldots \rightarrow \mathcal{E}_{1} \rightarrow \mathcal{J}_{X} \rightarrow 0
$$

where $s=\min \left\{k \geq c \mid H_{*}^{j}\left(\mathcal{J}_{X}\right)=0\right.$ for all $j$ with $\left.k+1 \leq j \leq n-k\right\}$ and $\mathcal{E}_{1}, \ldots, \mathcal{E}_{s}$ are Eilenberg-MacLane bundles which split as a direct sum of line bundles unless $\mathcal{E}_{i}$ has depth $2 i-1$ and $1 \leq i \leq v=\min \{n-c, s\}$.

For any such resolution it holds

$$
H_{*}^{i}\left(\mathcal{J}_{X}\right) \cong H_{*}^{2 i-1}\left(\mathcal{E}_{i}\right) \text { if } 1 \leq i \leq v .
$$

Proof. Consider the sequence $(+)$ of the ideal $I_{X}=H_{*}^{0}\left(\mathcal{J}_{X}\right)$ provided by Theorem 6.1. Due to the assumption on $X$ the modules $E_{1}, \ldots, E_{s}$ have cohomology of finite length. Thus the sheaves $\mathcal{E}_{i}=\tilde{E}_{i}$ are locally free and we obtain the sequence $(*)$ as sheafification of $(+)$.

Conversely, taking global sections in any sequence $(*)$ we get a sequence of the form $(+)$ as in Theorem 6.1. Thus the uniqueness properties of $(+)$ show that the sequence $(*)$ is indeed a locally free resolution.

The final assertion follows by tracing the cohomology along short exact sequences.

We want to give a name to a certain type of exact sequences.

Definition 6.5. A subscheme $X \subset \mathbb{P}^{n}$ of codimension $c$ is said to have a weak $\Omega$-resolution if there exists an exact sequence

$$
\begin{aligned}
0 \longrightarrow \mathcal{F}_{s} \stackrel{\alpha_{s}}{\longrightarrow} \ldots \stackrel{\alpha_{v+2}}{\longrightarrow} \mathcal{F}_{v+1} \longrightarrow \mathcal{F}_{v} \oplus \bigoplus_{j}\left(\Omega_{\mathbb{P}^{n}}^{2 v-1}\left(-e_{v . j}\right)\right)^{s_{v . j}} \stackrel{\alpha_{v}}{\longrightarrow} \\
\quad \ldots \stackrel{\alpha_{2}}{\longrightarrow} \mathcal{F}_{1} \oplus \bigoplus_{j}\left(\Omega_{\mathbb{P}^{n}}^{1}\left(-e_{1 . j}\right)\right)^{s_{1 . j}} \longrightarrow I \longrightarrow 0
\end{aligned}
$$

where $s=\min \left\{k \geq c \mid H_{*}^{j}\left(\mathcal{J}_{X}\right)=0\right.$ for all $j$ with $\left.k+1 \leq j \leq n-k\right\}, v=$ $\min \{n-c, s\}, \mathcal{F}_{1}, \ldots, \mathcal{F}_{s}$ are direct sums of line bundles and for fixed $i$ the integers $e_{i . j}$ are pairwise distinct.

The weak $\Omega$-resolution is called minimal if there is no line bundle $\mathcal{L}$ in the sequence such that the restriction of $\alpha_{i}(2 \leq i \leq s)$ to $\mathcal{L}$ induces an isomorphism of $\mathcal{L}$ onto $\mathcal{L}$.

Subschemes having a weak $\Omega$-resolution can be characterized cohomologically.

TheOREM 6.6. Let $X \subset \mathbb{P}^{n}$ be a subscheme of codimension $c$. Let $s=\min \{k \geq$ $c \mid H_{*}^{j}\left(\mathcal{J}_{X}\right)=0$ for all $j$ with $\left.k+1 \leq j \leq n-k\right\}$ and $v=\min \{n-c, s\}$. Then the following conditions are equivalent:

(a) It holds

$$
\mathfrak{m} \cdot H_{*}^{i}\left(\mathcal{J}_{X}\right)=0 \quad \text { if } 1 \leq i \leq v .
$$


(b) $X$ admits a weak $\Omega$-resolution

$$
\begin{aligned}
0 \rightarrow \mathcal{F}_{s} \rightarrow \ldots \rightarrow \mathcal{F}_{v+1} \rightarrow \mathcal{F}_{v} \oplus \bigoplus_{j}\left(\Omega_{\mathbb{P}^{n}}^{2 v-1}\left(-e_{v . j}\right)\right)^{s_{v . j}} \rightarrow \\
\quad \ldots \rightarrow \mathcal{F}_{1} \oplus \bigoplus_{j}\left(\Omega_{\mathbb{P}^{n}}^{1}\left(-e_{1 . j}\right)\right)^{s_{1 . j}} \rightarrow I \rightarrow 0 \\
\quad
\end{aligned}
$$

where $s_{i . j}=h^{i}\left(\mathcal{J}_{X}(j)\right)$.

Moreover, the weak $\Omega$-resolution is a locally free resolution, i.e., the minimal weak $\Omega$-resolution of $X$ is uniquely determined.

Proof. Recall that $\widetilde{G_{i+1}} \cong \Omega_{\mathbb{P}^{n}}^{i}$ if $R=K\left[x_{0}, \ldots, x_{n}\right]$ is a polynomial ring. Thus, the result follows by the Corollaries 6.3 and 6.4

REMARK 6.7. In Theorem 1.3 of the introduction we gave a characterization of quasi-Buchsbaum subschemes $X \subset \mathbb{P}^{n}$ where $c \geq \frac{n}{2}$. This result is is covered by Theorem 6.6 because $s=c$ and $v=n-c$ if $c \geq \frac{n}{2}$.

\section{Surfaces in $\mathbb{P}^{4}$}

Now we want to specialize some results to surfaces of codimension two. This gives a new prospect on the construction of smooth surfaces in $\mathbb{P}^{4}$. Then we derive a criterion on a weak $\Omega$-resolution of a surface $X$ in order to obtain a characterization of the arithmetically Buchsbaum surfaces among the quasi-Buchsbaum surfaces. This criterion could be extended to arbitrary quasi-Buchsbaum subschemes $X \subset \mathbb{P}^{n}$ such that $\operatorname{dim} X \leq \operatorname{codim} X$. We won't pursue this here. Instead, we explain how the criterion can be applied.

Proposition 7.1. Let $X \subset \mathbb{P}^{4}$ be a 2-dimensional subscheme. Then $X$ has a presentation

$$
0 \rightarrow \mathcal{E}_{2} \stackrel{\varphi}{\longrightarrow} \mathcal{E}_{1} \rightarrow \mathcal{J}_{X} \rightarrow 0
$$

where $\mathcal{E}_{2}$ and $\mathcal{E}_{1}$ are Eilenberg-MacLane sheaves such that $H_{*}^{i}\left(\mathcal{E}_{1}\right)=0$ if $i=2,3$ and $H_{*}^{i}\left(\mathcal{E}_{2}\right)=0$ if $i=1,2$.

If $\varphi$ is a minimal morphism, i.e. it does not map a line bundle summand of $\mathcal{E}_{2}$ onto a line bundle summand of $\mathcal{E}_{1}$, then the presentation is uniquely determined (up to isomorphisms of exact sequences).

Furthermore, the sheaf $\mathcal{E}_{1}$ is torsion-free, $\mathcal{E}_{2}$ is reflexive and

$$
H_{*}^{1}\left(\mathcal{E}_{1}\right) \cong H_{*}^{1}\left(\mathcal{J}_{X}\right) \text { and } H_{*}^{2}\left(\mathcal{E}_{2}\right) \cong H_{*}^{2}\left(\mathcal{J}_{X}\right) \text {. }
$$

Moreover, we have:

(a) $X$ is equidimensional if and only if $\mathcal{E}_{1}$ is a vector bundle and $\operatorname{dim} H_{*}^{3}\left(\mathcal{E}_{2}\right)^{\vee} \leq$ 1 .

(b) $X$ is equidimensional and Cohen-Macaulay if and only if $\mathcal{E}_{1}$ and $\mathcal{E}_{2}$ are vector bundles.

Proof. The existence of the asserted presentation is a consequence of Theorem 6.1. Since $\operatorname{dim} H_{*}^{1}\left(\mathcal{J}_{X}\right)^{\vee} \leq 1$ and $\operatorname{dim} H_{*}^{2}\left(\mathcal{J}_{X}\right)^{\vee} \leq 2$, Proposition 2.5 shows that $\mathcal{E}_{1}$ is torsion-free and that $\mathcal{E}_{2}$ is reflexive.

Claim (b) is a special case of Corollary 6.4. Claim (a) follows by combining Proposition 2.5 and the cohomological characterization of equidimensionality [26], Lemma 2.11. 
REMARK 7.2. (i) Using Horrocks' 16 characterization of stable equivalence classes of vector bundles on $\mathbb{P}^{4}$ Bolondi [4 also obtained the presentation of the statement in the case of equidimensional Cohen-Macaulay surfaces. But he did not establish its uniqueness property.

(ii) Using the Eagon-Northcott complex we may think of our subscheme $X$ as the degeneracy locus of the map $\varphi$. Note that the idea of constructing surfaces as degeneracy locus of vector bundles has been systematically exploited in $[8$. There the bundles are chosen in accordance with Beilinson's spectral sequence and not necessarily Eilenberg-MacLane sheaves.

(iii) The presentation above allows to construct an equidimensional surface with prescribed cohomology. Indeed, the cohomology of $X$ determines the cohomology of the Eilenberg-MacLane sheaves $\mathcal{E}_{1}$ and $\mathcal{E}_{2}$ which in turn determine $\mathcal{E}_{1}$ and $\mathcal{E}_{2}$ itself up to direct sums of line bundles according to Proposition 2.6. Then we have to choose a sufficiently general map $\varphi: \mathcal{E}_{2} \rightarrow \mathcal{E}_{1}$. If the degeneracy locus has codimension two then it is the desired surface.

Now we want to compare quasi-Buchsbaum and arithmetically Buchsbaum surfaces. Let $X \subset \mathbb{P}^{4}$ be a quasi-Buchsbaum surface. According to Theorem 6.6 it has a weak $\Omega$-resolution

$$
0 \rightarrow \mathcal{F}_{2} \oplus \bigoplus_{j}\left(\Omega_{\mathbb{P}^{4}}^{3}\left(-e_{2 . j}\right)\right)^{s_{2 . j}} \stackrel{\varphi}{\longrightarrow} \mathcal{F}_{1} \oplus \bigoplus_{j}\left(\Omega_{\mathbb{P}^{4}}^{1}\left(-e_{1 . j}\right)\right)^{s_{1 . j}} \rightarrow \mathcal{J}_{X} \rightarrow 0
$$

where $\mathcal{F}_{1}, \mathcal{F}_{2}$ are direct sums of line bundles on $\mathbb{P}^{4}$. Let $\mathcal{P}$ be a direct sum of line bundles such that there is an epimorphism $\delta: \mathcal{P} \rightarrow \mathcal{F}_{1} \oplus \bigoplus_{j}\left(\mathcal{O}_{\mathbb{P}^{4}}^{1}\left(-e_{1 . j}\right)\right)^{s_{1 . j}}$. Using this notation we can distinguish between quasi-Buchsbaum and arithmetically Buchsbaum subschemes as follows.

Proposition 7.3. The surface $X$ is arithmetically Buchsbaum if and only if there is a morphism $\alpha: \mathcal{F}_{2} \oplus \bigoplus_{j}\left(\Omega_{\mathbb{P}^{4}}^{3}\left(-e_{2 . j}\right)\right)^{s_{2 . j}} \rightarrow \mathcal{P}$ such that the following diagram is commutative

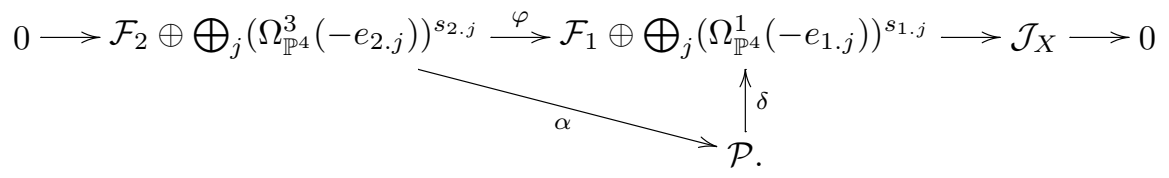

Proof. Assume that such a map $\alpha$ exists. Consider the exact commutative diagram

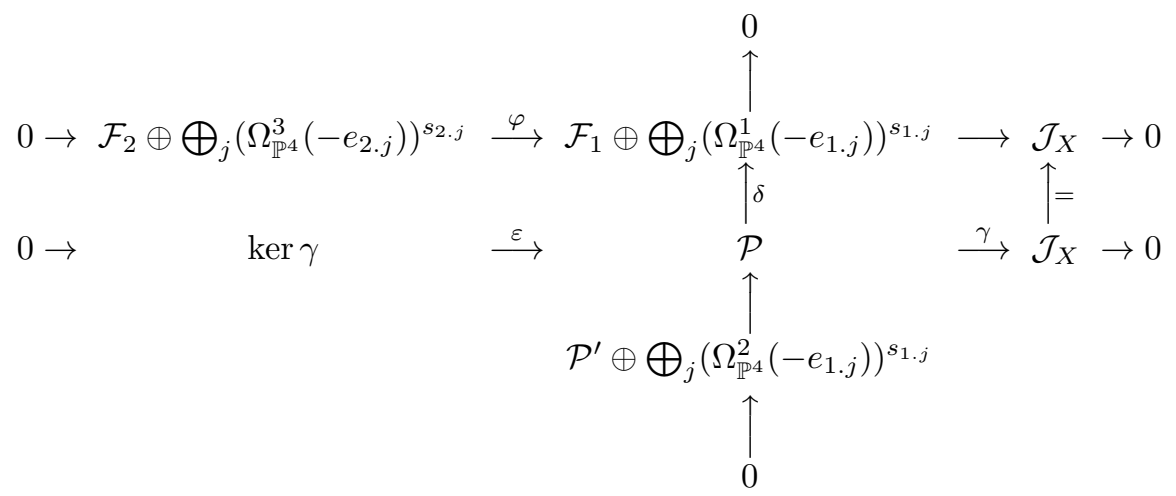


where $\mathcal{P}^{\prime}$ is a sum of line bundles. The Snake lemma provides the exact sequence

$$
0 \rightarrow \mathcal{P}^{\prime} \oplus \bigoplus_{j}\left(\Omega_{\mathbb{P}^{4}}^{2}\left(-e_{1 . j}\right)\right)^{s_{1 . j}} \rightarrow \operatorname{ker} \gamma \stackrel{\beta}{\longrightarrow} \mathcal{F}_{2} \oplus \bigoplus_{j}\left(\Omega_{\mathbb{P}^{4}}^{3}\left(-e_{2 . j}\right)\right)^{s_{2 . j}} \rightarrow 0 .
$$

This sequence splits because $\varepsilon^{-1} \circ \alpha$ is a splitting map. Indeed, our assumption implies $\operatorname{im} \alpha \subset \operatorname{ker} \gamma=\operatorname{im} \varepsilon$ and $\beta \circ\left(\varepsilon^{-1} \circ \alpha\right)$ is the identity map on $\mathcal{F}_{2} \oplus$ $\bigoplus_{j}\left(\Omega_{\mathbb{P}^{4}}^{3}\left(-e_{2 . j}\right)\right)^{s_{2 . j}}$. Therefore the second row of the first diagram implies that $X$ is arithmetically Buchsbaum according to Corollary 5.4 .

Now let us assume that $X$ is arithmetically Buchsbaum. Then $X$ has a locally free resolution

$$
0 \rightarrow \mathcal{F}_{2} \stackrel{\psi}{\longrightarrow} \mathcal{F}_{1}^{\prime} \oplus \bigoplus_{j}\left(\Omega_{\mathbb{P}^{4}}^{2}\left(-e_{2 . j}\right)\right)^{s_{2 . j}} \oplus \bigoplus_{j}\left(\Omega_{\mathbb{P}^{4}}^{1}\left(-e_{1 . j}\right)\right)^{s_{1 . j}} \rightarrow \mathcal{J}_{X} \rightarrow 0
$$

according to Corollary 5.4 .

Resolving $\bigoplus_{j}\left(\Omega_{\mathbb{P}^{4}}^{2}\left(-e_{2 . j}\right)\right)^{s_{2 . j}}$ and using the Snake lemma we get an exact commutative diagram

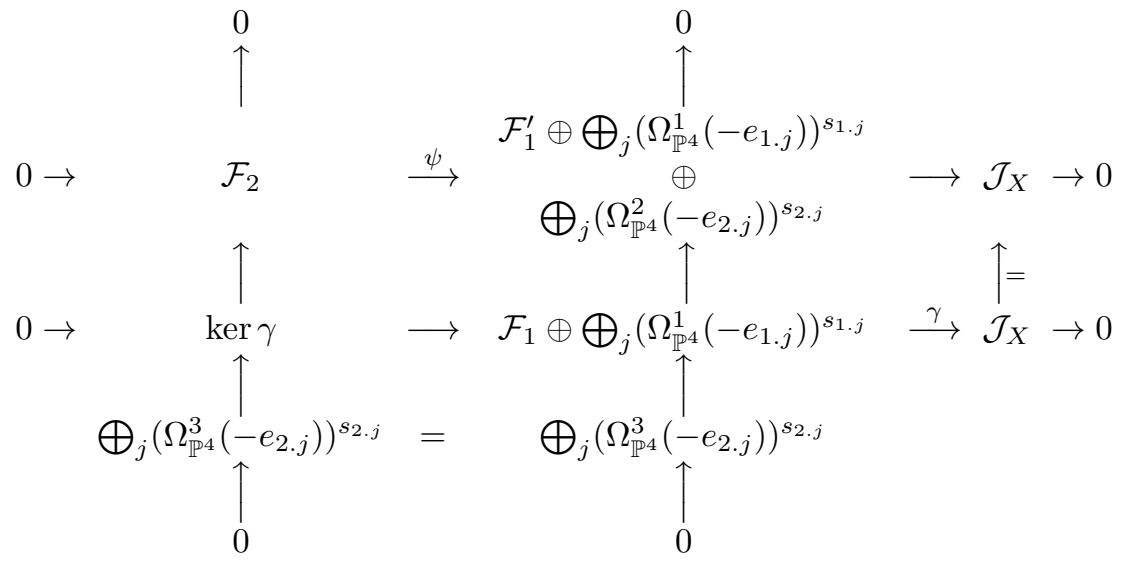

where $\mathcal{F}_{1}$ is a direct sum of line bundles. Since $\mathcal{F}_{2}$ is a direct sum of line bundles the vertical sequence on the left-hand side must split. Repeating the argument where we also resolve $\bigoplus_{j}\left(\Omega_{\mathbb{P}^{4}}^{1}\left(-e_{1 . j}\right)\right)^{s_{1 . j}}$ we get an exact commutative diagram

$$
\begin{aligned}
& 0 \rightarrow \mathcal{F}_{2} \oplus \bigoplus_{j}\left(\Omega_{\mathbb{P}^{4}}^{3}\left(-e_{2 . j}\right)\right)^{s_{2 . j}} \quad \stackrel{\psi^{\prime}}{\longrightarrow} \mathcal{F}_{1} \oplus \bigoplus_{j}\left(\Omega_{\mathbb{P}^{4}}^{1}\left(-e_{1 . j}\right)\right)^{s_{1 . j}} \rightarrow \mathcal{J}_{X} \rightarrow 0
\end{aligned}
$$



$$
\begin{aligned}
& \bigoplus_{j}\left(\Omega_{\mathbb{P}^{4}}^{2}\left(-e_{1 . j}\right)\right)^{s_{1 . j}}
\end{aligned}
$$

where $\pi$ is the canonical projection and $\mathcal{P}^{\prime}$ a direct sum of line bundles. Hence, denoting by $\alpha$ the restriction of $\psi^{\prime \prime}$ to $\mathcal{F}_{2} \oplus \bigoplus_{j}\left(\Omega_{\mathbb{P}^{4}}^{3}\left(-e_{2 . j}\right)\right)^{s_{2 . j}}$ we obtain the commutative diagram

$$
\begin{aligned}
& 0 \longrightarrow \mathcal{F}_{2} \oplus \bigoplus_{j}\left(\Omega_{\mathbb{P}^{4}}^{3}\left(-e_{2 . j}\right)\right)^{s_{2 . j}} \stackrel{\psi^{\prime}}{\longrightarrow} \mathcal{F}_{1} \oplus \bigoplus_{j}\left(\Omega_{\mathbb{P}^{4}}^{1}\left(-e_{1 . j}\right)\right)^{s_{1 . j}} \longrightarrow \mathcal{J}_{X} \longrightarrow 0 \\
& \longrightarrow \text {. } \uparrow_{\mathcal{F}_{1} \oplus \delta^{\prime}}
\end{aligned}
$$


This proves our claim for this particular weak $\Omega$-resolution and the particular epimorphism $\delta^{\prime}$. But splitting off redundant line bundles we get a minimal weak $\Omega$ resolution and a minimal epimorphism $\delta$ and thus our claim holds in this situation, too. Due to the uniqueness of minimal $\Omega$-resolutions and minimal free resolutions we can conclude that any weak $\Omega$-resolution admits a morphism $\alpha$ as claimed.

Now we want to show how the criterion above can be applied.

EXAmPLE 7.4. Every surface $X \subset \mathbb{P}^{4}$ with a weak $\Omega$-resolution

$$
0 \rightarrow\left(\Omega_{\mathbb{P}^{4}}^{3}(-1)\right)^{2} \stackrel{\varphi}{\longrightarrow} \mathcal{O}_{\mathbb{P}^{4}}(-4) \oplus\left(\Omega_{\mathbb{P}^{4}}^{1}(-3)\right)^{2} \rightarrow \mathcal{J}_{X} \rightarrow 0
$$

is quasi-Buchsbaum but not arithmetically Buchsbaum. Note that $X$ is a smooth rational surface of degree 10 if $\varphi$ is general enough (cf. [8], Example B1.15).

We need only to show that $X$ is not arithmetically Buchsbaum. Assuming the contrary there is by Proposition 7.3 a commutative diagram

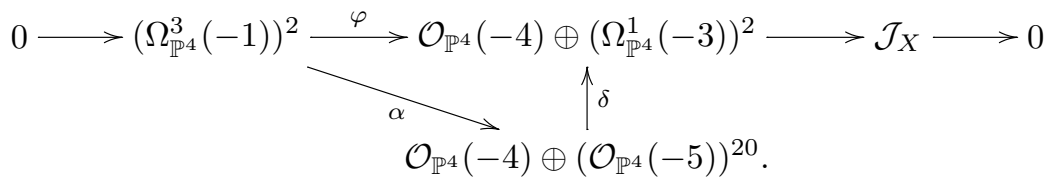

Since $\operatorname{Hom}_{\mathcal{O}^{4}}\left(\Omega_{\mathbb{P}^{4}}^{3}(-1), \mathcal{O}_{\mathbb{P}^{4}}(-5)\right) \cong H^{0}\left(\Omega_{\mathbb{P}^{4}}^{1}(-1)\right)=0$ we see that $\alpha$ must induce an injective map $\left(\Omega_{\mathbb{P}^{4}}^{3}(-1)\right)^{2} \rightarrow \mathcal{O}_{\mathbb{P}^{4}}(-4)$ which does not exist. This contradiction proves our claim.

EXAMPLE 7.5. Every surface $X \subset \mathbb{P}^{4}$ with a weak $\Omega$-resolution

$$
0 \rightarrow\left(\mathcal{O}_{\mathbb{P}^{4}}(-5)\right)^{2} \oplus \Omega_{\mathbb{P}^{4}}^{3}(-1) \stackrel{\varphi}{\longrightarrow}\left(\mathcal{O}_{\mathbb{P}^{4}}(-4)\right)^{3} \oplus \Omega_{\mathbb{P}^{4}}^{1}(-3) \rightarrow \mathcal{J}_{X} \rightarrow 0
$$

is quasi-Buchsbaum but not arithmetically Buchsbaum. Note that $X$ is a smooth elliptic surface of degree 10 if $\varphi$ is general enough (cf. [8], Example B7.5).

The proof that $X$ is not arithmetically Buchsbaum is similar to the one just given. Indeed, if $X$ were arithmetically Buchsbaum we had a map

$$
\alpha: \Omega_{\mathbb{P}^{4}}^{3}(-1) \rightarrow\left(\mathcal{O}_{\mathbb{P}^{4}}(-4)\right)^{3} \oplus\left(\mathcal{O}_{\mathbb{P}^{4}}(-5)\right)^{10}
$$

which must induce an injective map $\Omega_{\mathbb{P}^{4}}^{3}(-1) \rightarrow\left(\mathcal{O}_{\mathbb{P}^{4}}(-4)\right)^{3}$. Again, rank considerations show that such an embedding cannot exist.

\section{References}

[1] M. Amasaki, Application of the generalized Weierstrass preparation theorem to the study of homogeneous ideals, Trans. Amer. Math. Soc. 317 (1990), 1-43.

[2] A. Aure, W. Decker, K. Hulek, S. Popescu, K. Ranestad, Syzygies of abelian and bielliptic surfaces in $\mathbb{P}^{4}$, Internat. J. Math. 8 (1997), 849-919.

[3] M. Auslander, M. Bridger, Stable module theory, Mem. Amer. Math. Soc. 94, 1969.

[4] G. Bolondi, Surfaces in $\mathbb{P}^{4}$ and deficiency modules, Contemp. Math. 162 (1994), 49-63.

[5] W. Bruns, J. Herzog, "Cohen-Macaulay rings", Cambridge Studies in Advanced Mathematics 39, Cambridge University Press, 1993.

[6] M. Chang, Characterization of arithmetically Buchsbaum subschemes of codimension 2 in $\mathbb{P}^{n}$, J. Differential Geom. 31 (1990), 323-341.

[7] N. Chiarli, S. Greco, U. Nagel, Regularity bounds for projective subschemes of codimension two, Kyoto J. Math. 40 (2000), 37-59.

[8] W. Decker, L. Ein, F. Schreyer, Construction of surfaces in $\mathbb{P}_{4}$, J. Algebraic Geom. 2 (1993), 185-237.

[9] D. Eisenbud, S. Gôto, Linear free resolutions and minimal multiplicity, J. Algebra 88 (1984), 89-133. 
[10] E. G. Evans, P. Griffith, Syzygies, Cambridge University Press, 1985.

[11] E. G. Evans, P. Griffith, Filtering cohomology and lifting vector bundles, Trans. Amer. Math. Soc. 289 (1985), 321 - 332.

[12] S. Goto, Maximal Buchsbaum modules over regular local rings and a structure theorem for generalized Cohen-Macaulay modules, In: Advanced Studies in Pure Mathematics 11, 39-63, Kinokuniya-North Holland, 1987.

[13] M. Green, Koszul cohomology and the geometry of projective varieties, J. Differential Geom. 19 (1984), 125-171.

[14] M. Green, R. Lazarsfeld On the projective normality of complete linear series on an algebraic curve, Invent. Math. 83 (1986), 73-90.

[15] L. T. Hoa, C. Miyazaki, Bounds on the Castelnuovo-Mumford regularity for generalized Cohen-Macaulay graded rings, Math. Ann. 301 (1995), 587-598.

[16] G. Horrocks, Vector bundles on the punctured spectrum of a local ring, Proc. London Math. Soc. 14 (1964), 689-713.

[17] G. Horrocks, Construction of bundles on $\mathbb{P}^{n}$, Astérisque 71-72 (1980), 197-203.

[18] T. Kawasaki, Surjective-Buchsbaum modules over Cohen-Macaulay local rings, Math. Z. 218 (1995), 191-205.

[19] G. Kempf, Projective coordinate rings of abelian varieties, In: Algebraic analysis, geometry and number theory (ed. J. I. Igusa), 225-336, The John Hopkins Press, 1989.

[20] H. Lange, Ch. Birkenhake, Complex abelian varieties, Grundlehren der mathematischen Wissenschaften 302, Springer-Verlag, 1992.

[21] D. Mumford, Lectures on curves on an algebraic surface, Ann. of Math. Studies 59, Princeton Univ. Press, 1966.

[22] D. Mumford, Abelian varieties, Oxford University Press, 1974.

[23] P. Murthy, Generators for certain ideals in regular rings of dimension three, Comment. Math. Helv. 47 (1972), 179-184.

[24] U. Nagel, P. Schenzel, Degree bounds for generators of cohomology modules and Castelnuovo-Mumford regularity, Nagoya Math. J. 152 (1998), 153-174.

[25] U. Nagel, On arithmetically Buchsbaum subschemes and liaison, Habilitationsschrift, University of Paderborn, 1996.

[26] U. Nagel, Even liaison classes generated by Gorenstein linkage, J. Algebra 209 (1998), $543-584$.

[27] U. Nagel, Arithmetically Buchsbaum divisors on varieties of minimal degree, Trans. Amer. Math. Soc. 351 (1999), 4381-4409.

[28] G. Pareschi, Syzygies of abelian varieties, J. Amer. Math. Soc. 13 (2000), 651-664.

[29] A. P. Rao, Liaison among curves in $\mathbb{P}^{3}$, Invent. Math. 50 (1979), 205-217.

[30] J.-P. Serre, Sur le modules projectifs, Sem. Dubreil-Pisot 23 (1960/61), Expose 13.

[31] P. Schenzel, Dualisierende Komplexe in der lokalen Algebra und Buchsbaum-Ringe, LNM 907, Springer-Verlag, 1982.

[32] J. Stückrad, W. Vogel, Toward a theory of Buchsbaum singularities, Amer. J. Math. 100 (1978), 727-746.

[33] J. Stückrad, W. Vogel, Buchsbaum rings and applications, Springer-Verlag, 1986.

[34] W. Vogel, Über eine Vermutung von D. A. Buchsbaum, J. Algebra 25 (1973), 106-112.

[35] C. Walter, Transversality theorems in general characteristic and arithmetically Buchsbaum schemes, Internat. J. Math. 5 (1994), 609-617.

[36] K. Yamagishi, Bass number characterization of surjective Buchsbaum modules, Math. Proc. Cambridge Phil. Soc. 110 (1991), 261-279.

[37] Y. Yoshino, Maximal Buchsbaum modules of finite projective dimension, J. Algebra 159 (1993), 240-264

Fachbereich Mathematik und Informatik, Universität Paderborn, D-33095 PaderBORn, Germany

E-mail address: uwen@uni-paderborn.de

(Address since August 2002: Department of Mathematics, University of Kentucky, LeXington, KY 40506-0027, USA) 